\title{
SPIKES FOR THE GIERER-MEINHARDT SYSTEM WITH DISCONTINUOUS DIFFUSION COEFFICIENTS
}

\author{
JUNCHENG WEI AND MATTHIAS WINTER
}

\begin{abstract}
We rigorously prove results on spiky patterns for the Gierer-Meinhardt system [10] with a jump discontinuity in the diffusion coefficient of the inhibitor. Using numerical computations in combination with a Turing-type instability analysis, this system has been investigated by Benson, Maini and Sherratt [1], [3], [15].
\end{abstract}

Firstly, we show the existence of an interior spike located away from the jump discontinuity, deriving a necessary condition for the position of the spike. In particular we show that the spike is located in one-and-only-one of the two subintervals created by the jump discontinuity of the inhibitor diffusivity. This localization principle for a spike is a new effect which does not occur for homogeneous diffusion coefficients. Further, we show that this interior spike is stable.

Secondly, we establish the existence of a spike whose distance from the jump discontinuity is of the same order as its spatial extent. The existence of such a spike near the jump discontinuity is the second new effect presented in this paper.

To derive these new effects in a mathematically rigorous way, we use analytical tools like LiapunovSchmidt reduction and nonlocal eigenvalue problems which have been developed in our previous work $[33]$.

Finally, we confirm our results by numerical computations for the dynamical behavior of the system. We observe a moving spike which converges to a stationary spike located in the interior of one of the subintervals or near the jump discontinuity.

1991 Mathematics Subject Classification. Primary 35B35, 76E30; Secondary 35B40, 76E06.

Key words and phrases. Pattern Formation, Discontinuous diffusion coefficients, Steady states, Stability. 


\section{INTRODUCTION}

We consider a two-component Turing system [25] for which the first diffusion coefficient is constant (independent of the spatial variable), whereas the second has a jump discontinuity.

Systems with varying diffusion coefficients arise in biological or chemical modeling if a control chemical regulates the diffusion process of the morphogens in a reaction-diffusion system. Jump discontinuities of the diffusion coefficients play an important role, for example when two different tissues meet at a border. This border is often initiated by differing genetic expressions.

The effect most studied for reaction-diffusion systems is the Turing instability. For spatially homogeneous steady states, instabilities having an inhomogeneous spatial profile do occur, but spatially homogeneous instabilities are excluded. The fact that instabilities are necessarily varying in the spatial variable explains the onset of spatial patterns.

Turing in his pioneering paper [25] initiated this theory, studying a model for cell-to-cell interaction based on chemicals which he termed morphogens. In a closed spatially extended system for a certain choice of parameters, small fluctuations, which, for example, could be introduced by noise, are able to initiate pattern formation in the system. This process was later called diffusion-driven or Turing instability.

Since the paper of Turing, many reaction-diffusion equations have been introduced to model all kinds of biological effects leading to pattern formation such as animal skin patterns, wound healing, cancer growth, embryology, spread of epidemic diseases or genetic signaling pathways [19].

Most of these models use two species of chemicals and spatially homogeneous conditions (constant coefficients). The main reason for studying these systems is that even in this situation Turing instability may occur, and in many cases it can be studied analytically. Prominent examples of Turing systems are the Gierer-Meinhardt system [10], the Gray-Scott system [11], [12] and the Schnakenberg model [24].

For systems with piecewise constant diffusion coefficients, Turing instabilities have been computed numerically and investigated analytically by Benson, Maini and Sherratt [1], [3], [15], and results on dispersion relations and typical solution profiles have been obtained. In particular, the authors showed 
that the spatial variation of diffusion coefficients may produce isolated patterns and asymmetric spatially oscillating patterns which are not seen in standard homogeneous Turing systems.

Biological applications of these effects include explaining the anterior-posterior asymmetry of skeletal elements in limbs and experimental results on double anterior limbs [15], [36]. The fact that for asymmetric solutions different peaks may have different amplitudes is a possible explanation for the common observation that digits vary in length.

Prominent examples for which the genetic basis for the formation of a boundary is well understood are anterior-posterior (A-P) as well as dorsal-ventral (D-V) compartmentalization in the Drosophila wing imaginal disc or in the vertebrate brain. These effects have been reviewed in [4], [5], [14]. We give more details in the discussion section at the end of the paper.

To summarize, including spatial variation of the diffusion coefficients into reaction-diffusion systems is extremely well motivated by biological modeling and the new effects which are introduced by this generalization are commonly observed in biological applications.

We give a rigorous mathematical proof of the influence of discontinuous diffusion coefficients on the qualitative and quantitative properties of spiky patterns in a reaction-diffusion system.

In particular, in this paper we study the Gierer-Meinhardt system [10], which is one of the most popular Turing systems [25] of activator-inhibitor type. Adapted to our situation, it reads as follows:

$$
\left\{\begin{array}{l}
a_{t}=\epsilon^{2} a_{x x}-a+\frac{a^{2}}{h}, \\
\tau h_{t}=\left(D(x) h_{x}\right)_{x}-h+a^{2} .
\end{array}\right.
$$

Note that $h$ acts as an inhibitor to $a$, whereas $a$ acts as an activator to both $a$ and $h$. This motivates the name activator-inhibitor system. In this paper, we assume that $0<\epsilon<<1, \tau \geq \mathbf{0}$ are constants and

$$
D(x)= \begin{cases}D_{1}, & -1<x<x_{b}, \\ D_{2}, & x_{b}<x<1,\end{cases}
$$

where $0<D_{1}, 0<D_{2}$, and $D_{1} \neq D_{2}$. In Section 7 we set $\tau=0.1$ and in Section 13 we assume that $\tau$ is small enough. We study the equation $(1.1)$ in the interval $(-1,1)$ with Neumann boundary conditions

$$
a_{x}(-1)=a_{x}(1)=0, \quad h_{x}(-1)=h_{x}(1)=0
$$


This is the simplest case of environmental variation in diffusivity. It was observed in [3] that numerical solutions of models with more realistic forms for $D(x)$ give similar patterns to those computed when $D(x)$ is a step function. Therefore in this paper we focus on the case when $D(x)$ has one jump connecting two constant values.

We rigorously prove the existence of two classes of stationary spiky solutions to this system: first an interior spike which is located far away from the jump discontinuity and second a spike whose distance to the jump discontinuity is of the same order as its spatial extent.

A condition for the position of the interior spike will be derived, namely it can be located in oneand-only-one of the two subintervals. This localization principle for spikes is a new effect which does not occur for homogeneous diffusion coefficients. Further, we investigate the linearized stability of this interior spiky steady state and we prove that it is always stable.

Concerning the spike near the jump discontinuity, we will show that generically there are two possible locations or there is none. Further, our results imply that it occurs preferably if the discontinuity is located near the center of the interval and if the two diffusion constants differ by a substantial amount.

Now we mention some previous works on spiky steady states for the Gierer-Meinhardt system with constant coefficients. Existence and stability of spiky steady states have been studied for 1-D in [13] and their instabilities have been investigated in [27]. For 2-D the existence and stability of multiple spikes has been investigated in [31], [32], [33].

Works with spatially varying coefficients include [21], [22] where 1-D and 2-D complex patterns are investigated, [26] where the 1-D and 2-D motion of a spike and pinning effects are studied and [20] where the varied behavior of traveling pulses is shown.

The structure of the paper is as follows: in Section 2 we provide some preliminaries. In Section 3 we present the main results of the paper. In Section 4 we introduce and analyze a suitable approximate approximate solution to a spiky steady state. In Section 5 we summarize the proof of the stability of an interior spike. In Section 6 we show the existence of a steady state with a spike near the jump in the diffusion coefficient of the inhibitor. In Section 7 we confirm our analytical results by numerical 
computations. We also consider situations not analyzed in this paper, such as multi-spike solutions or two space dimensions. In Section 8 we discuss our results and put them into the biological context. In Appendix A (Section 9) we introduce and analyze the Green's function which is used throughout the paper. In Appendix B (Section 10) we derive some important estimates for the approximate steady state to an interior spike. In Appendix C (Section 11) we apply the method of Liapunov-Schmidt reduction to the system. In Appendix D (Section 12) we solve the reduced problem. In Appendix E (Section 13) we show the stability of the steady state with an interior spike.

\section{Preliminaries}

Before stating our main results in Section 3, in this section we introduce some notation and perform some preliminary analysis.

Throughout this paper, we always assume that $\Omega=(-1,1)$. With $L^{2}(\Omega)$ and $H^{2}(\Omega)$ we denote the usual Sobolev spaces.

Since we consider $(1.1)-(1.3)$ for a discontinuous diffusion coefficient $D(x)$ of the inhibitor $h$, which has a jump at a point $x_{b}$, the smoothness of $h$ at $x_{b}$ is lost. More precisely, for classical solutions $D(x) h_{x}(x)$ is continuous at $x=x_{b}$ and therefore $h_{x}(x)$ has a jump discontinuity at $x=x_{b}$. To account for this jump discontinuity of $h$ the function spaces have to be chosen very carefully.

We assume that

$$
(a, h) \in H_{N}^{2}(-1,1) \times H_{N}^{2, *}(-1,1)
$$

where

$$
\begin{aligned}
H_{N}^{2}(-1,1) & :=\left\{a \in H^{2}(-1,1): a_{x}(-1)=a_{x}(1)=0\right\}, \\
H^{2, *}(-1,1) & :=\left\{h \in H^{1}(-1,1):\left(D(x) h_{x}\right)_{x} \in L^{2}(-1,1)\right\}, \\
H_{N}^{2, *}(-1,1) & :=\left\{h \in H^{2, *}(-1,1): h_{x}(-1)=h_{x}(1)=0\right\},
\end{aligned}
$$

endowed with the norm

$$
\|(a, h)\|_{2, *}^{2}:=\|a\|_{H^{2}(-1,1)}^{2}+\|h\|_{2, *}^{2}, \text { where }\|h\|_{2, *}^{2}:=\|h\|_{H^{1}(-1,1)}^{2}+\left\|\left(D(x) h_{x}\right)_{x}\right\|_{L^{2}(-1,1)} .
$$


The variable $w$ denotes the unique homoclinic solution of the following problem:

$$
\begin{cases}w^{\prime \prime}-w+w^{2}=0 & \text { in } R^{1}, \\ w>0, \quad w(0)=\max _{y \in R} w(y), & w(y) \rightarrow 0 \text { as }|y| \rightarrow \infty .\end{cases}
$$

Note that $w$ is an even function and $w^{\prime}(y)<0$ if $y>0$. An explicit representation is

$$
w(y)=\frac{3}{2} \cosh ^{-2} \frac{y}{2} .
$$

We set

$$
\rho(y):=\int_{0}^{y} w^{2}(z) d z
$$

Elementary calculations give

$$
\begin{gathered}
\alpha:=\int_{0}^{\infty} w^{2}(y) d y=\int_{0}^{\infty} w(y) d y=3, \quad \int_{0}^{\infty} w^{3}(y) d y=3.6 \\
\rho(y)=\frac{9}{2} \tanh \frac{y}{2}-\frac{3}{2} \tanh ^{3} \frac{y}{2}, \quad \int_{0}^{\infty} w^{3}(y) \rho(y) d y=\frac{297}{64}=\mathbf{4 . 6 4 0 6 2 5} \\
\int_{0}^{\infty}\left(w^{\prime}\right)^{2} d y=\int_{0}^{\infty} w^{3} d y-\int_{0}^{\infty} w^{2} d y=0.6 .
\end{gathered}
$$

To conclude this section, we study a nonlocal linear operator. We first recall the following result.

Theorem 2.1. [30] Consider the nonlocal eigenvalue problem

$$
L \phi:=\Delta \phi-\phi+2 w \phi-\gamma \frac{\int_{R} w \phi d y}{\int_{R} w^{2} d y} w^{2}=\lambda \phi, \quad \phi \in H^{1}(R) .
$$

(1) If $\gamma<1$, then there is a positive eigenvalue to (2.4).

(2) If $\gamma>1$, then for any nonzero eigenvalue $\lambda$ of (2.4) we have

$$
\operatorname{Re}(\lambda) \leq-c_{0}<0 \quad \text { for some } c_{0}>0
$$

(3) If $\gamma \neq 1$ and $\lambda=0$, then

$$
\phi=c_{0} \frac{d w}{d y}
$$

for some constant $c_{0}$.

The conjugate operator of $L$ under the scalar product in $L^{2}(R)$ is

$$
L^{*} \psi=\Delta \psi-\psi+2 w \psi-\gamma \frac{\int_{R} w^{2} \psi d y}{\int_{R} w^{2} d y} w, \quad H^{2}(R) \rightarrow L^{2}(R) .
$$


Then we have the following result.

Lemma 2.2. (Lemma 3.2 of [34].) If $\gamma \neq 1$, then

$$
X_{0}:=\operatorname{Ker}\left(L^{*}\right)=\operatorname{span}\left\{\frac{d w}{d y}\right\} .
$$

As a consequence, we have

Lemma 2.3. The operator

$$
L: H^{2}(R) \rightarrow L^{2}(R)
$$

restricted to the spaces

$$
L: X_{0}^{\perp} \cap H^{2}(R) \rightarrow X_{0}^{\perp} \cap L^{2}(R),
$$

where the $X_{0}^{\perp}$ denotes the orthogonal projection with respect to the scalar product of $L^{2}(R)$, is invertible. Moreover, $L^{-1}: X_{0}^{\perp} \cap L^{2}(R) \rightarrow X_{0}^{\perp} \cap H^{2}(R)$ is bounded.

Proof: This follows from the Fredholm Alternative Theorem and Lemma 2.2.

3. MAIN RESUlTS: interior SPIKE AND SPIKE NEAR THE JUMP DISCONTINUITY OF THE DIFFUSION COEFFICIENT

We consider the case when the inhibitor diffusivity is discontinuous with a single jump, and derive two types of one-spike steady states:

1. an interior spike located far away from the jump discontinuity of the inhibitor diffusivity (see Theorem 3.1). For this interior spike we derive a new localization principle, which states that the spike can exist in one-and-only-one of the two sub-intervals divided by the jump discontinuity. Further, we show that this solution is stable (Theorem 3.3).

2. a spike near the jump discontinuity whose center has a distance of order $\epsilon$ from the jump discontinuity, which means that its distance from the jump discontinuity is of the same order as the spatial extent of the spike (Theorem 3.4). 
We re-scale $\Omega_{\epsilon}=\left(-\frac{1}{\epsilon}, \frac{1}{\epsilon}\right)$ and define $u(x) \in H_{\epsilon}^{2}(\Omega)$ if and only if $u\left(\frac{x}{\epsilon}\right) \in H^{2}\left(\Omega_{\epsilon}\right)$, where the norm of the former space is defined by the norm of the latter, i.e.

$$
\|u\|_{H_{\epsilon}^{2}(\Omega)}:=\|u(\cdot / \epsilon)\|_{H^{2}\left(\Omega_{\epsilon}\right)} .
$$

In the same way we introduce this re-scaling to the other function spaces introduced at the beginning of the previous section.

Now we state our first main theorem.

Theorem 3.1. (Existence of an interior-spike solution.) Suppose that the condition

$$
\frac{1}{\theta_{1}} \tanh \theta_{1}\left(1+x_{b}\right)>\frac{1}{\theta_{2}} \tanh \theta_{2}\left(1-x_{b}\right)
$$

holds, where $\theta_{i}=D_{i}^{-1 / 2}$. Then there exists a steady state of (1.1) - (1.3) with an interior spike for the activator which is located in the subinterval $\left(-1, x_{b}\right)$. More precisely, we have

$$
a_{\epsilon}(x) \sim \xi_{0} w\left(\frac{x-t^{\epsilon}}{\epsilon}\right)+o(1) \quad \text { in } H_{\epsilon}^{2}(\Omega)
$$

where $t^{\epsilon} \rightarrow t_{0} \in\left(-1, x_{b}\right)$ and $\xi_{0} / h\left(t^{\epsilon}\right) \rightarrow 1$ as $\epsilon \rightarrow 0$. The limit position $t_{0}$ is given by

$$
\frac{1}{\theta_{1}} \tanh \left(\theta_{1}\left(2 t_{0}+1-x_{b}\right)\right)=\frac{1}{\theta_{2}} \tanh \left(\theta_{2}\left(1-x_{b}\right)\right) \text {. }
$$

If (3.1) holds then there do not exist any steady states of (1.1) - (1.3) with an interior spike for the activator in the subinterval $\left(x_{b}, 1\right)$.

Remark 3.2. (i) Condition (3.1) of Theorem 3.1 implies that in the case $x_{b}=0$, i.e. if the jump discontinuity is located at the center of the interval, there exists a spike in the subinterval with the larger diffusion constant $D_{1}$ (and the smaller $\theta_{1}$ ) but not in the other subinterval. This follows from the fact that the function $\tanh \alpha / \alpha$ is strictly monotone decreasing for $\alpha>\mathbf{0}$.

(ii) Condition (3.1) combines the effects of sub-domain size and diffusion constant. Hence the localization effect is due jointly, and favorably, to relatively large subinterval and large diffusion constant.

(iii) Note that existence of a single spike occurs in one-and-only-one of the subintervals.

(iv) The reverse sign of (3.1) does not have to be studied separately. It follows by reflection about the center $x=0$ of the interval. By this transformation $\theta_{1}$ and $\theta_{2}$ are exchanged and the sign of $x_{b}$ is 
reversed. An easy calculation shows that the inequality resulting from this transformation is equivalent to (3.1) with reversed sign.

(v) The localization principle proved in Theorem 3.1 which has been established here for a reactiondiffusion system with a jump discontinuity of the inhibitor diffusivity is expected to play a general role for reaction-diffusion systems with varying diffusion coefficients. We expect that further investigations of this phenomenon in the general context will show important new effects which are not observed in the classical case of Turing systems with constant diffusion coefficients. This should have important implications for the prediction of localization and asymmetry of patterns in many areas of biology.

We now give a result for the linear stability of the interior spike.

Theorem 3.3. (Stability of an interior-spike solution.) The interior spike established in Theorem 3.1 is linearly stable.

In our second main theorem we establish the existence of spikes near the jump discontinuity of the inhibitor diffusivity, more precisely at a distance of order $\epsilon$ from this discontinuity.

Theorem 3.4. (Existence of spikes near the jump discontinuity $x_{b}$ of the inhibitor diffusivity.)

(i) If

$$
\left\{\begin{array}{l}
\theta_{1}<\theta_{2} \quad \text { and } \\
0<\frac{\theta_{2} \tanh \theta_{1}\left(1+x_{b}\right)-\theta_{1} \tanh \theta_{2}\left(1-x_{b}\right)}{\theta_{2} \tanh \theta_{1}\left(1+x_{b}\right)+\theta_{1} \tanh \theta_{2}\left(1-x_{b}\right)}<\frac{\theta_{2}^{2}-\theta_{1}^{2}}{2 \theta_{1}^{2}} \frac{I\left(L_{0}\right)}{10.8},
\end{array}\right.
$$

there exist exactly two spikes near the jump discontinuity $x_{b}$. They are given by (3.2) with $t^{\epsilon}=x_{b}-\epsilon L$ for two possible values of $L$ which are solutions of (6.7).

(ii) If condition (3.1) holds and $\theta_{1}>\theta_{2}$, or if

$$
\left\{\begin{array}{l}
\theta_{1}<\theta_{2} \quad \text { and } \\
\frac{\theta_{2} \tanh \theta_{1}\left(1+x_{b}\right)-\theta_{1} \tanh \theta_{2}\left(1-x_{b}\right)}{\theta_{2} \tanh \theta_{1}\left(1+x_{b}\right)+\theta_{1} \tanh \theta_{2}\left(1-x_{b}\right)}>\frac{\theta_{2}^{2}-\theta_{1}^{2}}{2 \theta_{1}^{2}} \frac{I\left(L_{0}\right)}{10.8}>0,
\end{array}\right.
$$

there is no spike near the jump discontinuity $x_{b}$. More precisely, there is no spike given by (3.2) with $\left|t^{\epsilon}-x_{b}\right|=O(\epsilon)$ 
In Theorem 3.4 we have used

$$
I(L):=\int_{L}^{\infty} w^{3}(y)(\rho(y)-\beta) d y
$$

where

$$
\beta=\alpha \frac{\theta_{2} \tanh \theta_{1}\left(1+x_{b}\right)-\theta_{1} \tanh \theta_{2}\left(1-x_{b}\right)}{\theta_{2} \tanh \theta_{1}\left(x_{b}+1\right)+\theta_{1} \tanh \theta_{2}\left(1-x_{b}\right)},
$$

$\rho(y)$ is given by (2.2), $L_{0}$ is uniquely determined by $\rho\left(L_{0}\right)=\beta$, and $\alpha=3$.

Remark 3.5. (i) We have the following results for the positions of the two spikes given by Theorem 3.4 (i) with respect to the jump discontinuity:

both are positive if

$$
\frac{\theta_{2} \tanh \theta_{1}\left(1+x_{b}\right)-\theta_{1} \tanh \theta_{2}\left(1-x_{b}\right)}{\theta_{2} \tanh \theta_{1}\left(1+x_{b}\right)+\theta_{1} \tanh \theta_{2}\left(1-x_{b}\right)}>\mathbf{0 . 4 2 9 6 8 7 5} \frac{\theta_{2}^{2}-\theta_{1}^{2}}{\theta_{2}^{2}+\theta_{1}^{2}}
$$

one is positive and the other is negative if

$$
0<\frac{\theta_{2} \tanh \theta_{1}\left(1+x_{b}\right)-\theta_{1} \tanh \theta_{2}\left(1-x_{b}\right)}{\theta_{2} \tanh \theta_{1}\left(1+x_{b}\right)+\theta_{1} \tanh \theta_{2}\left(1-x_{b}\right)}<\mathbf{0 . 4 2 9 6 8 7 5} \frac{\theta_{2}^{2}-\theta_{1}^{2}}{\theta_{2}^{2}+\theta_{1}^{2}} .
$$

Note that positive position ( $L>0)$ means that the spike is located to the left of the jump discontinuity, and negative position $(L<0)$ means that it lies to the right of the jump discontinuity.

(ii) We do not prove stability of the spike near the jump. However, our conjecture is that the spike at the left position is unstable and the spike at the right position is stable.

We have the following simple nonexistence result for spikes near the jump discontinuity.

Corollary 3.6. Suppose that $\theta_{1}<\theta_{2}$ and

$$
\left|\frac{\theta_{2} \tanh \theta_{1}\left(1+x_{b}\right)-\theta_{1} \tanh \theta_{2}\left(1-x_{b}\right)}{\theta_{2} \tanh \theta_{1}\left(1+x_{b}\right)+\theta_{1} \tanh \theta_{2}\left(1-x_{b}\right)}\right|>\mathbf{0 . 4 2 9 6 8 7 5} \frac{\theta_{2}^{2}-\theta_{1}^{2}}{2 \theta_{1}^{2}} .
$$

Then there is no spike near the jump discontinuity, i.e. a spike with the center satisfying $\left|t^{\epsilon}-x_{b}\right|=$ $O(\epsilon)$.

Remark 3.7. The criterion given in Corollary 3.6 for nonexistence is satisfied if $\theta_{1}$ and $\theta_{2}$ are fixed constants which satisfy $\theta_{1}<\theta_{2}<\mathbf{2 . 3 7 7 9 3} \theta_{1}$ and the jump location $x_{b}$ is sufficiently close to +1 or -1. This means that we have non-existence if the diffusion constants are sufficiently close to each other and the jump discontinuity is located sufficiently close to either end of the interval. 


\section{THE approximate steady state to an interior spike}

We now construct an approximate steady state of (1.1) - (1.3) which has a spike of the activator $a(x)$ concentrating at a point $t_{0} \in(-1,1) \backslash\left\{x_{b}\right\}$. For $t_{0} \in(-1,1)$, let $t \in B_{\epsilon^{3 / 4}}\left(t_{0}\right):=$ $\left\{t \in(-1,1):\left|t-t_{0}\right|<\epsilon^{3 / 4}\right\}$. Note that if $t_{0} \neq x_{b}$, then for $\epsilon$ small enough we have $x_{b} \notin B_{\epsilon^{3 / 4}}\left(t_{0}\right)$. Set

$$
w_{0}(x)=w\left(\frac{x-t}{\epsilon}\right)
$$

where $w(y)$ is given by $(2.1)$. Let $r_{0}$ be such that

$$
r_{0}=\frac{1}{10}\left(\min \left(t_{0}+1,1-t_{0}\right)\right) \text {. }
$$

Introduce a smooth cut-off function $\chi: R \rightarrow[0,1]$ such that

$$
\chi(x)=1 \text { for }|x|<1 \text { and } \chi(x)=0 \text { for }|x|>2 .
$$

Setting

$$
\tilde{w}_{0}(x)=w_{0}(x) \chi\left(\frac{x-t}{r_{0}}\right)
$$

then $\tilde{w}_{0}(x)$ satisfies

$$
\epsilon^{2} \Delta \tilde{w}_{0}-\tilde{w}_{0}+\tilde{w}_{0}^{2}+\text { e.s.t. }=0 \quad \text { in }(-1,1), \quad \tilde{w}_{0}^{\prime}(-1)=\tilde{w}_{0}^{\prime}(1)=0
$$

where "e.s.t." denotes exponentially small terms. For $t \in(-1,1)$, let

$$
\hat{\xi}_{0}(t)=\frac{1}{G(t, t)},
$$

where $G(x, y)$ is the Green's function, defined in (9.1). It can be used to represent a steady state of the second equation of (1.1) and plays a major role throughout the paper. This Green's function will

be analyzed in detail in Appendix A. We will mostly drop the argument of $\hat{\xi}_{0}(t)$ and write $\hat{\xi}_{0}$ instead. Set

$$
\xi_{0}:=\hat{\xi}_{0} \xi_{\epsilon}
$$

where

$$
\xi_{\epsilon}:=\left(\epsilon \int_{R} w^{2}(z) d z\right)^{-1}=\frac{1}{6 \epsilon}
$$

Then, finally, we choose the first component of our approximate steady state for (1.1) to be

$$
w_{\epsilon, t}(x)=\xi_{0} \tilde{w}_{0}(x) .
$$


For a function $A \in L^{2}(-1,1)$, we define $T[A]$ to be the solution in $\mathbf{H}_{\mathbf{N}}^{\mathbf{2}, *}(-\mathbf{1}, \mathbf{1})$ of

$$
\left(D(x)(T[A])_{x}\right)_{x}-T[A]+A^{2}=0 .
$$

By standard elliptic theory, the solution $T[A]$ is positive and unique.

For $A=w_{\epsilon, t}$, where $t \in B_{\epsilon^{3 / 4}}\left(t_{0}\right)$, we choose the function $T[A](x)$ to be the second component of our approximate steady state for (1.1).

Some error estimates for the approximate steady state $\left(w_{\epsilon, t}, T\left[w_{\epsilon, t}\right]\right)$ are required. They will be derived in Appendix B and form the basis of the existence proof. In Appendix $\mathrm{C}$ we will reduce the problem to finite dimensions by Liapunov-Schmidt reduction. In Appendix D we will solve the resulting finite-dimensional problem.

We now discuss some observations about the limit positions of the interior spike by analyzing (3.3).

For the solution of (3.3) we have $t_{0} \rightarrow 0$ in either of the limits

(i) $\theta_{2} \rightarrow \theta_{1}$ (for $\theta_{1}=$ const.) (the system approaches the standard GM system with constant diffusivities).

(ii) $\theta_{1} \rightarrow 0$ and $\theta_{2} \rightarrow 0$ (the system approaches the shadow system with $D=\infty$ ).

The spike connects to the spike in the center of the domain, which is the unique spiky steady state for constant diffusivities or the shadow system.

(iii) In the limit $\theta_{2} \rightarrow \infty$ (for $\theta_{1}=$ const.) we get $t_{0} \rightarrow\left(x_{b}-1\right) / 2 \in\left(-1, x_{b}\right)$. Note that $-1<\left(x_{b}-1\right) / 2<0$. The spikes moves to the center of the subinterval with finite diffusion constant $D_{1}$ of the inhibitor if the other diffusion constant $D_{2}$ tends to zero.

(iv) Finally, if $\theta_{1}$ and $\theta_{2}$ are such that the inequality (3.1) approaches equality, the limit position $t_{0}$ approaches $x_{b}$.

Note that we always have $t_{0} \geq\left(x_{b}-1\right) / 2$ since otherwise (3.3) can not hold $\left(t_{0}<\left(x_{b}-1\right) / 2\right.$ implies l.h.s. is negative while r.h.s. is positive). 
By the above, we observe that we can obtain a single spike steady state centered at any point of the open interval $\left(\left(x_{b}-1\right) / 2, x_{b}\right)$ by varying the diffusion constants $D_{1}$ and $D_{2}$. This is a new effect which does not occur for constant diffusivities, where the interior spike is always located at the center of the interval. This shows that for discontinuous diffusion coefficients asymmetric instead of symmetric spike positions are the commonplace. This has some important consequences for biological applications, e.g. for understanding limb development.

\section{Stability of the interior spike}

We now indicate the main steps of the proof that the interior spike steady state is linearly stable. The small $(o(1))$ and large $(O(1))$ eigenvalues as $\epsilon \rightarrow 0$ are studied separately. It is easy to see that there are no eigenvalues which tend to infinity - we omit the proof of this statement, see e.g. [33].

For large eigenvalues one has to study nonlocal eigenvalue problems. For small eigenvalues one has to apply a projection to a finite-dimensional space similar to Liapunov-Schmidt reduction. The details of the stability proofs will be given in Appendix E.

\section{SPIKE NEAR THE JUMP DISCONTINUITY OF THE INHIBITOR DIFFUSIVITY}

We prove Theorem 3.4 on the existence of spikes near the jump discontinuity $\mathbf{x}_{\mathbf{b}}$ of the inhibitor diffusivity.

Proof of Theorem 3.4: Let

$$
a_{\epsilon}(x)=\xi_{0} w\left(\frac{x-t^{\epsilon}}{\epsilon}\right) \chi\left(\frac{x-t^{\epsilon}}{\epsilon}\right)+O(\epsilon) \text { in } H^{2}\left(\Omega_{\epsilon}\right),
$$

where $t_{\epsilon}$ is the center of the spike, $x_{b}-t^{\epsilon}=\epsilon L$ and $\xi_{0}$ is given by (4.7). Then we compute an approximation to the second component $h_{\epsilon}(x)$. We decompose $h_{\epsilon}$ into two parts:

$$
h_{\epsilon}(x)=\xi_{0}\left(\epsilon h_{1}\left(\frac{x-t^{\epsilon}}{\epsilon}\right)+h_{2}(x)\right)+O(\epsilon) \text { in } H^{2, *}\left(\Omega_{\epsilon}\right),
$$

where the inner expansion $h_{1}(y)$ for $y=\left(x-t^{\epsilon}\right) / \epsilon$ satisfies

$$
\left\{\begin{array}{l}
\left(D\left(t^{\epsilon}+\epsilon y\right) h_{1, y}(y)\right)_{y}+w^{2}(y)=0, \\
h_{1}(0)=0, h_{1, y}(0)=0
\end{array}\right.
$$


and the outer expansion $h_{2}(x)$ is given by

$$
\left\{\begin{array}{l}
\left(D(x) h_{2, x}(x)\right)_{x}-h_{2}(x)-\epsilon h_{1}(x)=0 \\
h_{2, x}( \pm 1)=-h_{1, y}( \pm \infty)
\end{array}\right.
$$

\section{Integrating (6.2) yields}

$$
h_{1, y}(y)= \begin{cases}-\theta_{1}^{2} \rho(y), & -\infty<y<L, \\ -\theta_{2}^{2} \rho(y), & L<y<\infty,\end{cases}
$$

where $\theta_{i}=D_{i}^{-1 / 2}$ and $\rho(y)$ has been defined in (2.2).

Recalling from (2.3) that

$$
\alpha=\int_{\mathbf{0}}^{\infty} w^{2}(z) d z=3
$$

we have

$$
h_{1, y}(-\infty)=\theta_{1}^{2} \alpha, \quad h_{1, y}(\infty)=-\theta_{2}^{2} \alpha
$$

Note that (2.3) implies

$$
\frac{\int_{0}^{\infty} w^{3}(y) \rho(y) d y}{\alpha \int_{0}^{\infty} w^{3}(y)}=\mathbf{0 . 4 2 9 6 8 7 5}
$$

Integrating (6.4) again, we have (up to order $O(\epsilon)$ which is included into the error term in (6.1))

$$
\epsilon h_{1}\left(\frac{x-t^{\epsilon}}{\epsilon}\right)= \begin{cases}\theta_{1}^{2} \alpha\left(x-x_{b}\right), & -1<x<x_{b}, \\ -\theta_{2}^{2} \alpha\left(x-x_{b}\right), & x_{b}<x<1 .\end{cases}
$$

Hence $h_{2}$ satisfies (up to order $O(\epsilon)$ which is included into the error term in (6.1))

$$
\left\{\begin{array}{l}
\left(D(x) h_{2, x}(x)\right)_{x}-h_{2}(x)-\epsilon h_{1}(x)=0 \\
h_{2, x}(-1)=-\theta_{1}^{2} \alpha, \quad h_{2, x}(1)=\theta_{2}^{2} \alpha
\end{array}\right.
$$

Solving (6.6), using (6.5), we get

$$
h_{2}(x)=\left\{\begin{array}{lc}
-\theta_{1}^{2} \alpha\left(x-x_{b}\right)+A \theta_{1} \frac{\cosh \theta_{1}(x+1)}{\cosh \theta_{1}\left(x_{b}+1\right)}, & -1<x<x_{b}, \\
\theta_{2}^{2} \alpha\left(x-x_{b}\right)+B \theta_{1} \frac{\cosh \theta_{2}(x-1)}{\cosh \theta_{2}\left(x_{b}-1\right)}, & x_{b}<x<1,
\end{array}\right.
$$

Continuity of the function $h_{2}(x)$ at $x=x_{b}$ gives $A=B$ and continuity of $D(x) h_{2, x}(x)$ at $x=x_{b}$ implies

$$
0=D_{1} h_{2, x}\left(x_{b}^{-}\right)-D_{2} h_{2, x}\left(x_{b}^{+}\right)=A\left(\tanh \theta_{1}\left(x_{b}+1\right)+\frac{\theta_{1}}{\theta_{2}} \tanh \theta_{2}\left(1-x_{b}\right)\right)-2 \alpha
$$

and so we have

$$
A=\frac{2 \alpha \theta_{2}}{\theta_{2} \tanh \theta_{1}\left(x_{b}+1\right)+\theta_{1} \tanh \theta_{2}\left(1-x_{b}\right)}
$$


(Note that (3.1) implies

$$
A>\frac{\alpha}{\tanh \theta_{1}\left(x_{b}+1\right)}
$$

Hence

$$
D_{1} h_{2, x}\left(x_{b}^{-}\right)=D_{2} h_{2, x}\left(x_{b}^{+}\right)=A \tanh \theta_{1}\left(x_{b}+1\right)-\alpha=\alpha \frac{\theta_{2} \tanh \theta_{1}\left(x_{b}+1\right)-\theta_{1} \tanh \theta_{2}\left(1-x_{b}\right)}{\theta_{2} \tanh \theta_{1}\left(x_{b}+1\right)+\theta_{1} \tanh \theta_{2}\left(1-x_{b}\right)}
$$

which implies

$$
h_{2, x}\left(x_{b}^{-}\right)=\theta_{1}^{2} \beta, \quad h_{2, x}\left(x_{b}^{+}\right)=\theta_{2}^{2} \beta,
$$

where

$$
\beta=\alpha \frac{\theta_{2} \tanh \theta_{1}\left(x_{b}+1\right)-\theta_{1} \tanh \theta_{2}\left(1-x_{b}\right)}{\theta_{2} \tanh \theta_{1}\left(x_{b}+1\right)+\theta_{1} \tanh \theta_{2}\left(1-x_{b}\right)} .
$$

Finally, we apply Liapunov-Schmidt reduction as in Appendix $\mathbf{C}$ to reduce the problem to one dimension. Following the argument in Appendix D to solve this reduced problem and determine the position of the spike we get the following solvability condition:

$$
\begin{aligned}
0 & =\xi_{\epsilon}^{-1} \int_{-\infty}^{\infty} w^{3}(y) h_{x}\left(t^{\epsilon}+\epsilon y\right) d y+O(\epsilon) \\
& =\int_{-\infty}^{\infty} w^{3}(y)\left(h_{1, y}(y)+h_{2, x}\left(t^{\epsilon}+\epsilon y\right)\right) d y+O(\epsilon) \\
& =\int_{-\infty}^{L} w^{3}(y)\left(-\theta_{1}^{2} \rho(y)+h_{2, x}\left(x_{b}^{-}\right)\right) d y+\int_{L}^{\infty} w^{3}(y)\left(-\theta_{2}^{2} \rho(y)+h_{2, x}\left(x_{b}^{+}\right)\right) d y+O(\epsilon) \\
& =\theta_{1}^{2}\left(\int_{-\infty}^{L} w^{3}(y)(-\rho(y)+\beta) d y\right)+\theta_{2}^{2}\left(\int_{L}^{\infty} w^{3}(y)(-\rho(y)+\beta) d y\right)+O(\epsilon) \\
& =\theta_{1}^{2}\left(\int_{-\infty}^{\infty} w^{3}(y)(-\rho(y)+\beta) d y-\int_{L}^{\infty} w^{3}(y)(-\rho(y)+\beta) d y\right)+\theta_{2}^{2}\left(\int_{L}^{\infty} w^{3}(y)(-\rho(y)+\beta) d y\right)+O(\epsilon) \\
& =\beta \theta_{1}^{2} \int_{-\infty}^{\infty} w^{3}(y) d y+\left(\theta_{2}^{2}-\theta_{1}^{2}\right) \int_{L}^{\infty} w^{3}(y)(-\rho(y)+\beta) d y+O(\epsilon)
\end{aligned}
$$

since $\rho(y)$ is an odd function.

Hence, for given $\theta_{1}, \theta_{2}, \beta$, we need to find $L$ such that

$$
\beta \theta_{1}^{2} \int_{-\infty}^{\infty} w^{3}(y) d y+\left(\theta_{2}^{2}-\theta_{1}^{2}\right) \int_{L}^{\infty} w^{3}(y)(-\rho(y)+\beta) d y=0 .
$$

We recall and summarize that

$$
\beta=\alpha \frac{\theta_{2} \tanh \theta_{1}\left(1+x_{b}\right)-\theta_{1} \tanh \theta_{2}\left(1-x_{b}\right)}{\theta_{2} \tanh \theta_{1}\left(1+x_{b}\right)+\theta_{1} \tanh \theta_{2}\left(1-x_{b}\right)}, \quad \alpha=3, \quad \rho(y)=\int_{0}^{y} w^{2}(z) d z=\frac{9}{2} \tanh \frac{y}{2}-\frac{3}{2} \tanh ^{3} \frac{y}{2} .
$$

We now check when condition (6.7) can be satisfied. We need to consider only the case $\beta>0$ which is equivalent to (3.1). Note that for $\beta=0$ (6.7) is not possible if $\theta_{1} \neq \theta_{2}$ and so we do not consider that case any further. 
The case $\beta<0$ can be reduced to the case $\beta>0$ by reflection about the center $x=0$ of the domain. This can be seen as follows: by this reflection $\theta_{1}$ and $\theta_{2}$ are exchanged, $x_{b}, t^{\epsilon}, \beta$ all change sign. Note that the order of the locations of the jump discontinuity and the spike are reversed so that the equation $x_{b}=t^{\epsilon}+\epsilon y$ with $y=L$ changes to $-x_{b}=-t^{\epsilon}+\epsilon y$ with $y=-L$. As a result, (6.7) is transformed to

$$
-\beta \theta_{2}^{2} \int_{-\infty}^{\infty} w^{3}(y) d y+\left(\theta_{1}^{2}-\theta_{2}^{2}\right) \int_{-L}^{\infty} w^{3}(y)(-\rho(y)+\beta) d y=0
$$

which is equivalent to $(6.7)$.

We know from Theorem 3.1 that the interior spike solution must be located to the left of the jump discontinuity. Now we show that generically for the spike near the jump discontinuity there are two possible locations or there is none.

A necessary condition is

$$
\theta_{1}^{2}<\theta_{2}^{2}
$$

Otherwise (6.7) implies

$$
\beta \theta_{1}^{2} \int_{-\infty}^{L} w^{3}(y) d y+\theta_{1}^{2} \int_{L}^{\infty} w^{3}(y) \rho(y) d y=\theta_{2}^{2} \int_{L}^{\infty} w^{3}(y)(\rho(y)-\beta) d y .
$$

If $\theta_{1}^{2} \geq \theta_{2}^{2}$ in the last equation we have l.h.s is greater than r.h.s. which gives a contradiction.

We now study (6.7) in detail.

An important observation is that the integrand of

$$
\int_{L}^{\infty} w^{3}(y)(-\rho(y)+\beta) d y
$$

changes sign at $\rho(y)=\beta$.

The function $\rho$ has the following properties:

$$
\begin{gathered}
\rho(0)=0, \quad \rho^{\prime}(y)=w^{2}(y)>0, \quad \rho(-y)=-\rho(y), \\
\rho(y) \rightarrow \int_{0}^{\infty} w^{2} d y=\alpha(=3) \text { as } y \rightarrow \infty
\end{gathered}
$$

and $\beta$ satisfies the inequality

$$
0<\beta=\alpha \frac{\theta_{2} \tanh \theta_{1}\left(1+x_{b}\right)-\theta_{1} \tanh \theta_{2}\left(1-x_{b}\right)}{\theta_{2} \tanh \theta_{1}\left(x_{b}+1\right)+\theta_{1} \tanh \theta_{2}\left(1-x_{b}\right)}<\alpha .
$$


Thus for all $0<\beta<\alpha$ there is exactly one positive $y=: L_{0}>0$ such that $\rho\left(L_{0}\right)-\beta=0$. Further, $\rho(y)-\beta<0$ if $0<y<L_{0}$ and $\rho(y)-\beta>0$ if $y>L_{0}$.

To give an explicit formula for $L_{0}$, using (2.3) we compute

$$
\rho\left(L_{0}\right)=\frac{9}{2} \tanh \frac{L_{0}}{2}-\frac{3}{2} \tanh ^{3} \frac{L_{0}}{2}=\beta .
$$

From this equation $L_{0}$ can be uniquely calculated.

Recall from (3.6) that for any real number $L$ we have defined

$$
I(L):=\int_{L}^{\infty} w^{3}(y)(\rho(y)-\beta) d y
$$

Then

(i) $I(L) \rightarrow 0$ as $L \rightarrow \infty, \quad I(L) \rightarrow-7.2 \beta<0$ as $L \rightarrow-\infty$,

(ii) $I(L)$ achieves its unique maximum among all real $L$ at $L=L_{0}>0$, where $I\left(L_{0}\right)>0$,

(iii) $I(L)$ is monotone increasing on $\left(-\infty, L_{0}\right)$,

(iv) $I(L)$ is monotone decreasing on $\left(L_{0}, \infty\right)$,

(v) $I(L)=0$ for a unique $L=L_{1}<0$.

Therefore the equation $I(L)=c$ has

$$
\begin{cases}\text { two solutions } & \text { if } 0<c<I\left(L_{0}\right), \\ \text { one solution } & \text { if } c=I\left(L_{0}\right) \text { or }-7.2 \beta<c \leq 0, \\ \text { no solution } & \text { if } c>I\left(L_{0}\right) \text { or } c \leq-7.2 \beta\end{cases}
$$

Since we assume $\theta_{1}<\theta_{2}$, for (6.7) only the case $c>0$ is relevant. Combining (6.9) with (6.7) and putting $c=\frac{\theta_{2} \tanh \theta_{1}\left(1+x_{b}\right)-\theta_{1} \tanh \theta_{2}\left(1-x_{b}\right)}{\theta_{2} \tanh \theta_{1}\left(1+x_{b}\right)+\theta_{1} \tanh \theta_{2}\left(1-x_{b}\right)} \frac{10.8 \cdot 2 \theta_{1}^{2}}{\theta_{2}^{2}-\theta_{1}^{2}}$, we have

(i) two solutions for (6.7) if

$$
0<\frac{\theta_{2} \tanh \theta_{1}\left(1+x_{b}\right)-\theta_{1} \tanh \theta_{2}\left(1-x_{b}\right)}{\theta_{2} \tanh \theta_{1}\left(1+x_{b}\right)+\theta_{1} \tanh \theta_{2}\left(1-x_{b}\right)}<\frac{\theta_{2}^{2}-\theta_{1}^{2}}{2 \theta_{1}^{2}} \frac{I\left(L_{0}\right)}{10.8} .
$$

(ii) no solution for (6.7) if

$$
\frac{\theta_{2} \tanh \theta_{1}\left(1+x_{b}\right)-\theta_{1} \tanh \theta_{2}\left(1-x_{b}\right)}{\theta_{2} \tanh \theta_{1}\left(1+x_{b}\right)+\theta_{1} \tanh \theta_{2}\left(1-x_{b}\right)}>\frac{\theta_{2}^{2}-\theta_{1}^{2}}{2 \theta_{1}^{2}} \frac{I\left(L_{0}\right)}{10.8} .
$$

The proof of Theorem 3.4 is completed. 
Proof of Remark 3.5: We compute

$$
I(0)=10.8\left(\mathbf{0 . 4 2 9 6 8 7 5}-\frac{\theta_{2} \tanh \theta_{1}\left(1+x_{b}\right)-\theta_{1} \tanh \theta_{2}\left(1-x_{b}\right)}{\theta_{2} \tanh \theta_{1}\left(1+x_{b}\right)+\theta_{1} \tanh \theta_{2}\left(1-x_{b}\right)}\right) .
$$

Therefore (6.7) implies that in Case (i) both solutions are positive if $c>I(0)$, which implies

$$
\frac{\theta_{2} \tanh \theta_{1}\left(1+x_{b}\right)-\theta_{1} \tanh \theta_{2}\left(1-x_{b}\right)}{\theta_{2} \tanh \theta_{1}\left(1+x_{b}\right)+\theta_{1} \tanh \theta_{2}\left(1-x_{b}\right)}>\mathbf{0 . 4 2 9 6 8 7 5} \frac{\theta_{2}^{2}-\theta_{1}^{2}}{\theta_{2}^{2}+\theta_{1}^{2}},
$$

one is positive, the other negative if $c<I(0)$, which implies

$$
0<\frac{\theta_{2} \tanh \theta_{1}\left(1+x_{b}\right)-\theta_{1} \tanh \theta_{2}\left(1-x_{b}\right)}{\theta_{2} \tanh \theta_{1}\left(1+x_{b}\right)+\theta_{1} \tanh \theta_{2}\left(1-x_{b}\right)}<\mathbf{0 . 4 2 9 6 8 7 5} \frac{\theta_{2}^{2}-\theta_{1}^{2}}{\theta_{2}^{2}+\theta_{1}^{2}}
$$

Proof of Corollary 3.6: We consider the following two limits. As $\beta \rightarrow 0$ we have $L_{0} \rightarrow 0$ and

$$
I\left(L_{0}\right) \rightarrow \int_{0}^{\infty} w^{3}(y) \rho(y) d y=4.640625
$$

As $\beta \rightarrow \alpha=3$ we have $L_{0} \rightarrow \infty$ and $I\left(L_{0}\right) \rightarrow 0$. For $\beta$ varying between these two extreme values the change of $I\left(L_{0}\right)$ is strictly monotone.

As a consequence we get $0 \leq I\left(L_{0}\right) \leq \mathbf{4 . 6 4 0 6 2 5}$ which implies the following necessary condition for existence:

$$
\mathbf{0}<\frac{\theta_{2} \tanh \theta_{1}\left(1+x_{b}\right)-\theta_{1} \tanh \theta_{2}\left(1-x_{b}\right)}{\theta_{2} \tanh \theta_{1}\left(1+x_{b}\right)+\theta_{1} \tanh \theta_{2}\left(1-x_{b}\right)}<\mathbf{0 . 4 2 9 6 8 7 5} \frac{\theta_{2}^{2}-\theta_{1}^{2}}{2 \theta_{1}^{2}} .
$$

Again, for $\beta<0$, we can use the reflection argument mentioned before. Then with the assumption $\theta_{1}>\theta_{2}$, we get the formula in Corollary 3.6.

Proof of Remark 3.7: There are cases when (6.10) is not true. For example, given $\theta_{1}, \theta_{2}$ such that

$$
0<\theta_{1}<\theta_{2}<\left(\frac{2.4296875}{\mathbf{0 . 4 2 9 6 8 7 5}}\right)^{\mathbf{1} / 2} \theta_{1} \approx 2.37793 \theta_{1}
$$

then

$$
\mathbf{0}<\mathbf{0 . 4 2 9 6 8 7 5} \frac{\theta_{2}^{2}-\theta_{1}^{2}}{2 \theta_{1}^{2}}<1
$$

and so by choosing $x_{b}$ close enough to 1 or -1 (6.10) fails to hold. So in this case there are no spikes in order $\epsilon$ distance from the jump discontinuity. The proof is finished. 
A spike approaching the jump discontinuity of the diffusion coefficient is a new phenomenon which to the best of our knowledge has not been observed, analyzed or discussed in the literature before.

We now discuss the behavior of spikes near the jump in certain limits.

(i) If $\theta_{2} \rightarrow \infty$ (and $\theta_{1}=$ const.), we have $\beta \rightarrow 3$. We can re-write (6.7) as

$$
7.2 \beta \frac{\theta_{1}^{2}}{\theta_{2}^{2}-\theta_{1}^{2}}=I(L)
$$

Solutions to (6.7) exist iff

$$
7.2 \beta \frac{\theta_{1}^{2}}{\theta_{2}^{2}-\theta_{1}^{2}}<I\left(L_{0}\right)
$$

An asymptotic analysis reveals that

$$
e^{-2 L_{0}} \sim c(3-\beta), \quad I\left(L_{0}\right) \sim c(3-\beta)^{5 / 2}
$$

and

$$
\frac{\theta_{1}}{\theta_{2}} \sim c(3-\beta)
$$

as $\beta \rightarrow 3$. Therefore in $(6.11)$ we have

$$
\text { l.h.s. } \sim c(3-\beta)^{2} \text { and r.h.s. } \sim c(3-\beta)^{5 / 2}
$$

and so (6.11) does not hold if $\beta$ is sufficiently close to 3 . For increasing $\beta$ at some threshold $\beta_{0}$ with $0<\beta<3$ both spike locations $L$ approach the same positive limit which is given by $L_{0}$ evaluated at $\beta_{0}$. For $\beta_{0}<\beta<3$ the spikes near the jump cease to exist.

(ii) If $x_{b} \rightarrow 1$, we get $\tanh \theta_{2}\left(1-x_{b}\right) \rightarrow 0$. This implies that we also have $\beta \rightarrow 3$. Repeating the previous analysis, we get

$$
e^{-2 L_{0}} \sim c(3-\beta), \quad I\left(L_{0}\right) \sim c(3-\beta)^{5 / 2}
$$

and

$$
\frac{\theta_{1}}{\theta_{2}} \sim c
$$

as $\beta \rightarrow 3$. Arguing similarly as in Case (i), we see that spikes near the jump cease to exist. In Corollary 3.6 and Remark 3.7 this argument has been made quantitative and explicit bounds for nonexistence have been derived. 
(iii) If $\beta \rightarrow 0$, from (6.7) we get

$$
\left(\theta_{2}^{2}-\theta_{1}^{2}\right) \int_{L}^{\infty} w^{3}(y) \rho(y) d y \rightarrow 0
$$

For $\theta_{2}^{2}-\theta_{1}^{2} \neq 0$, this implies that $L \rightarrow-\infty$ or $L \rightarrow \infty$. In this limit, the spikes at the jump connect to the interior spikes, which converge to the jump.

(iv) The case when $\beta \rightarrow 0$ and simultaneously $\theta_{2} \rightarrow \theta_{1}:=\theta$ requires further investigation. An example for this coincidence is given by $x_{b}=0$. Then (6.7) implies

$$
5.4[1+\theta(\tanh \theta-\operatorname{coth} \theta)]=\int_{L}^{\infty} w^{3}(y) \rho(y) d y .
$$

This equation has a solution iff

$$
5.4[1+\theta(\tanh \theta-\operatorname{coth} \theta)]<\int_{0}^{\infty} \mathbf{w}^{\mathbf{3}}(\mathbf{y}) \rho(\mathbf{y}) \mathbf{d} \mathbf{y}=4.640625 .
$$

It is an elementary computation to show that l.h.s. in (6.12) has the limit zero as $\theta \rightarrow 0$, is strictly monotone increasing in $\theta$ and has the limit 5.4 as $\theta \rightarrow \infty$. This implies that (6.7) has a solution iff $\theta$ is small enough. Then (6.7) has two solutions $L_{1}$ and $L_{2}$ with $L_{1}=-L_{2}$. These solutions are symmetric with respect to the jump discontinuity.

\section{Numerical COMPUtations}

We now show some numerical computations for the time-dependent behavior of system (1.1). We choose $\Omega=(-1,1), \tau=0.1$ and varying diffusion coefficients $\epsilon^{2}$. We divide $\Omega$ at either $x_{b}=0$ or $x_{b}=0.5$ and choose different constants for $D(x)$ on each of the resulting subintervals.

In each situation we present the solution for $t=10^{5}$. By this time the computation has come to a standstill in all cases, and this steady state is numerically stable (long-time limit). For the $1-D$ computations, the first component, $a$, is shown on the left and the second component, $h$, is displayed on the right. 
We first plot the initial conditions which are the same for all computations. We study the following two examples:
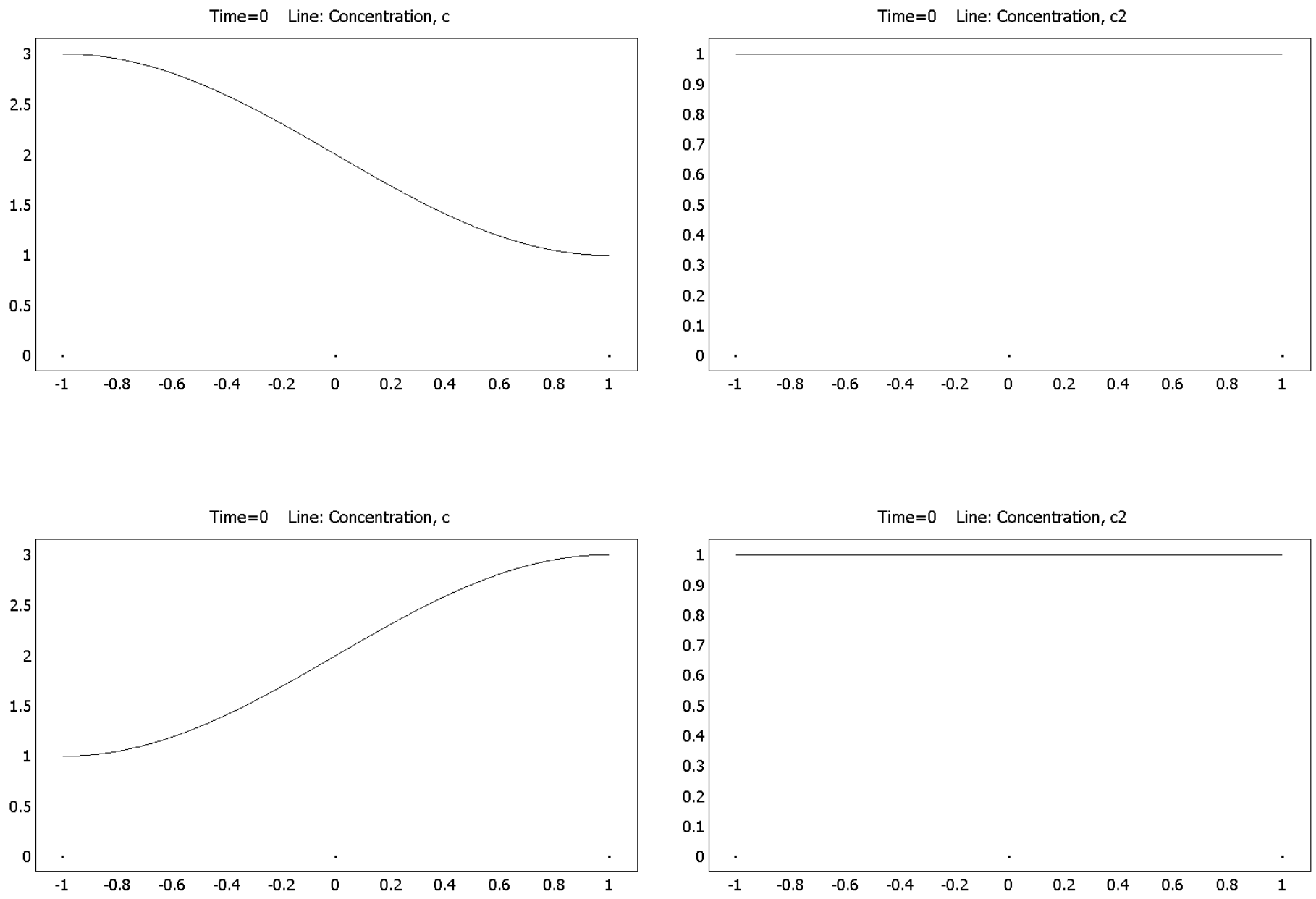

Figure 1. Initial condition for $a$ at $t=0$ : in the first example we take $a=2-\sin (x \pi / 2)$, i.e. the maximum is located at the left boundary. In the second example we take $a=2+\sin (x \pi / 2)$, i.e. the maximum is located at the right boundary. Initial condition for $h$ at $t=0: h=1$ for both examples. Both examples of initial conditions are investigated for all $1-D$ computations which follow. 
We now show a computation of a spike which either reaches a position near the jump discontinuity of the inhibitor diffusion (moving in from the left) or an interior position (moving in from the right) at $t=10^{5}$. The spike near the jump discontinuity is located slightly left of it which corresponds to the stable spike location having $L>0$.
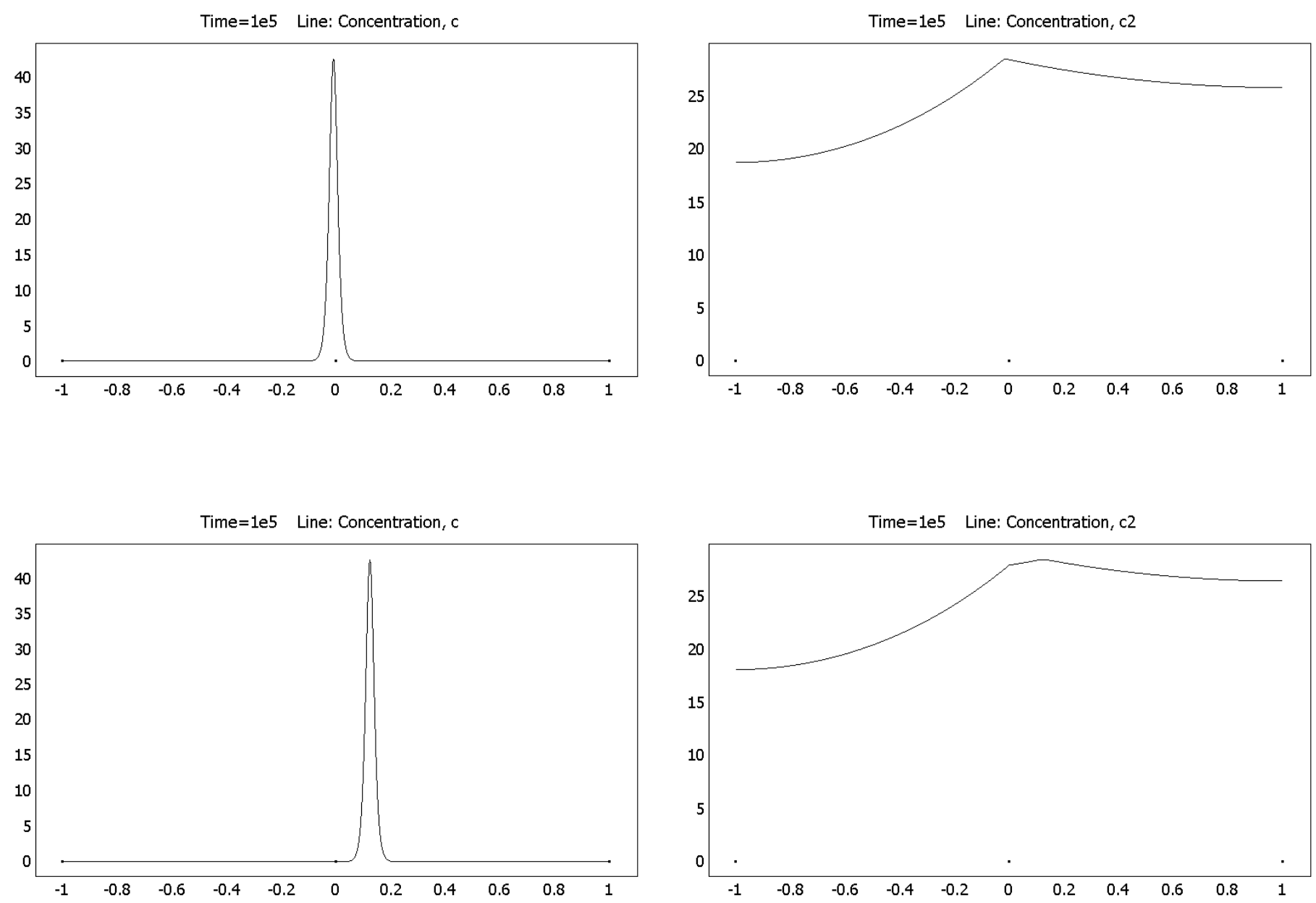

Figure 2. Long-time limit of the solution to (1.1) - (1.3) with $\epsilon^{2}=0.0001$ and $D(x)=1$ for $-1<x<0$, $D(x)=5$ for $0<x<1$. The initial conditions are given in Figure 1. We observe a spike near the jump discontinuity of the inhibitor diffusivity and a spike in the right subinterval. The conditions (3.1) and (3.4), respectively, are satisfied. Equation (3.3) implies $t_{0} \approx 0.10336$ for the position of the interior spike in the second example, which is in good agreement with the figure. 
Doing the computation with the same two initial conditions but changing the jump discontinuity of the diffusion coefficient of the inhibitor from $x_{b}=0.5$ to $x_{b}=0$ the result is similar. However, the limit position changes. In both examples the spike moves to the same interior spike which is located near the center $x=0$.
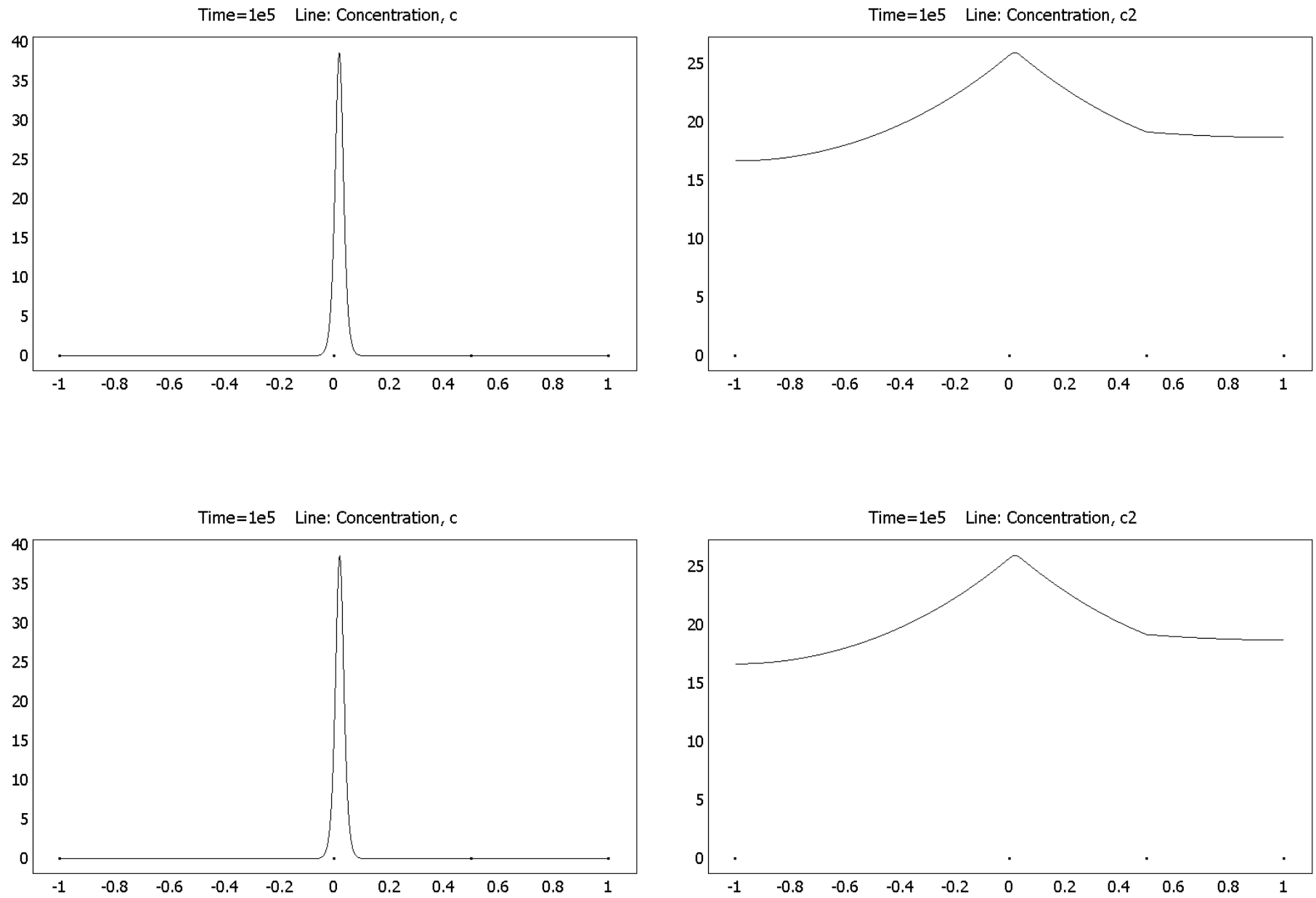

Figure 3. Long-time limit of the solution to (1.1) - (1.3) with $\epsilon^{2}=0.0001$ and $D(x)=1$ for $-1<x<0.5$, $D(x)=5$ for $0.5<x<1$. The initial conditions are given in Figure 1. We observe an interior spike in the left subinterval. The condition (3.1) is satisfied. Equation (3.3) implies $t_{0} \approx 0.01556$ for the position of the interior spike, which is in good agreement with the figure. 
Now we show the computations for some effects not analyzed in this paper. The initial conditions are again the two examples shown in Figure 1. We compute the following situation: $\epsilon^{2}=0.0001, D(x)=0.1$ for $-1<x<x_{b}, D(x)=0.5$ for $x_{b}<x<1$ for varying $x_{b}$. If we decrease $D(x)$ we expect solutions with multiple spikes. Some examples of this are shown in the following two figures.
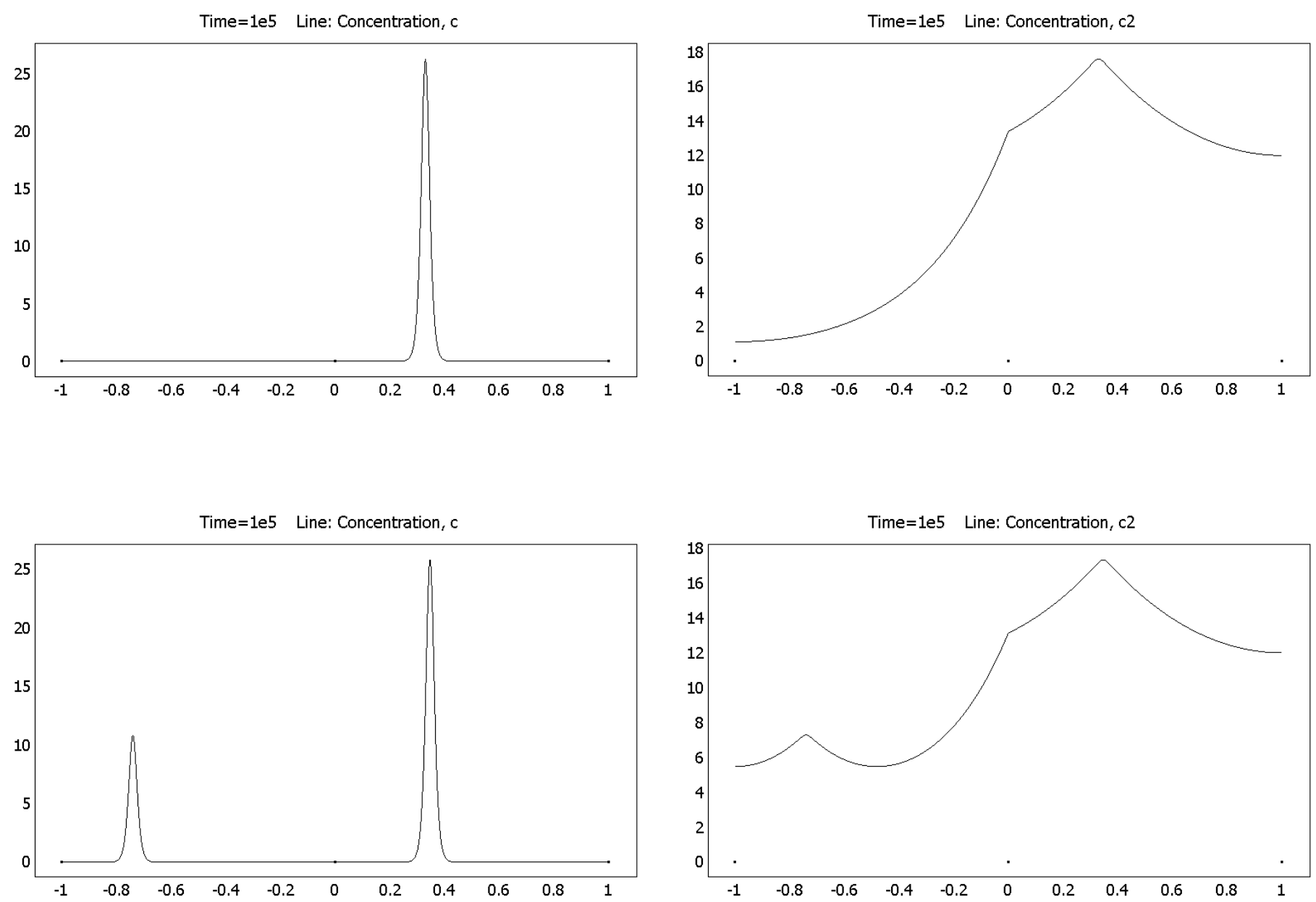

Figure 4. Long-time limit of the solution to (1.1) - (1.3) with $\epsilon^{2}=0.0001$ and $D(x)=0.1$ for $-1<x<0$, $D(x)=0.5$ for $0<x<1$. The initial conditions are given in Figure 1 . We observe an interior spike in the right subinterval or two interior spikes in different subintervals. Equation (3.3) implies $t_{0} \approx 0.33057$ for the position of the interior spike in the first example, which is in good agreement with the figure. 
Time $=1$ e5 Line: Concentration, $\mathrm{c}$

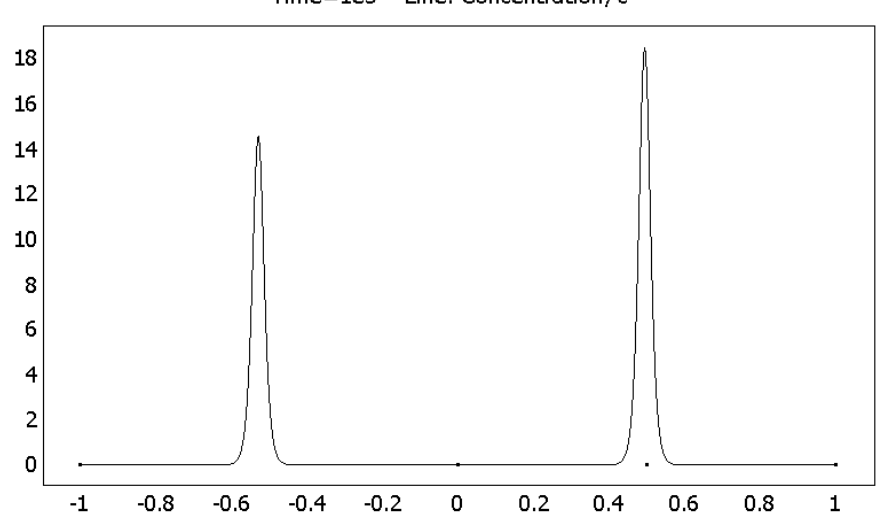

Time $=1$ e5 Line: Concentration, $c$

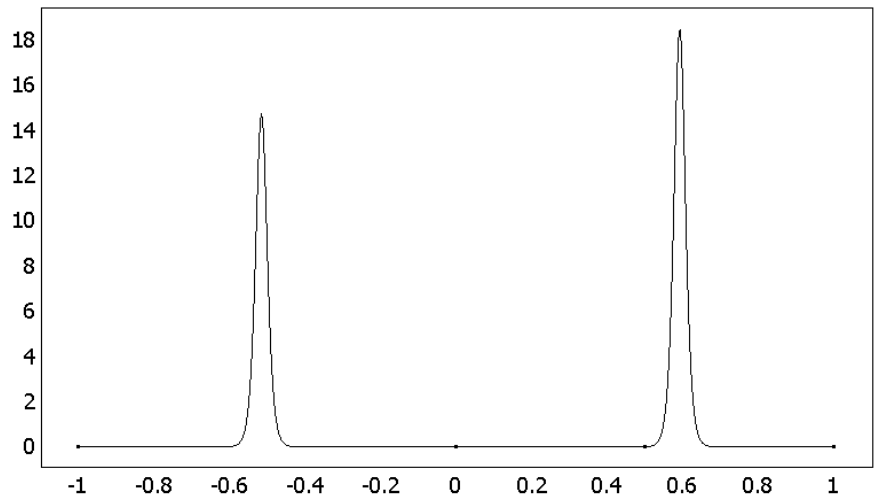

Time $=1$ e5 Line: Concentration, c2

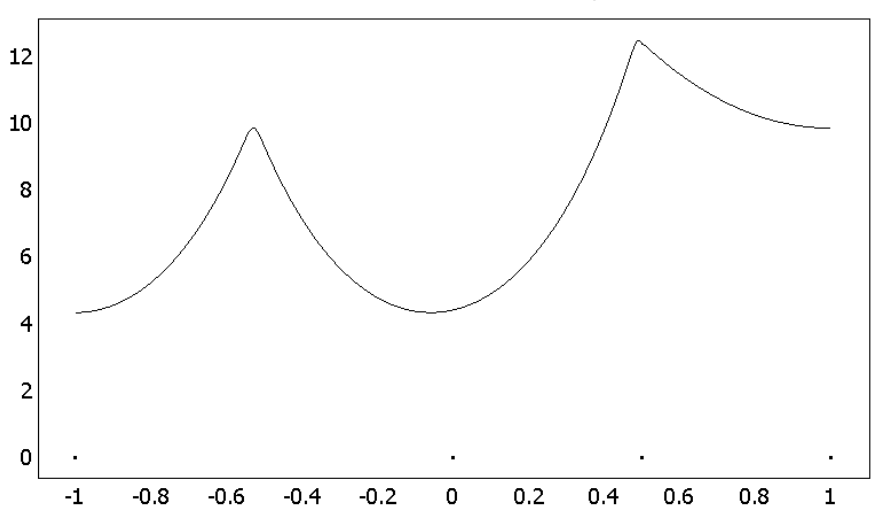

Time=1e5 Line: Concentration, c2

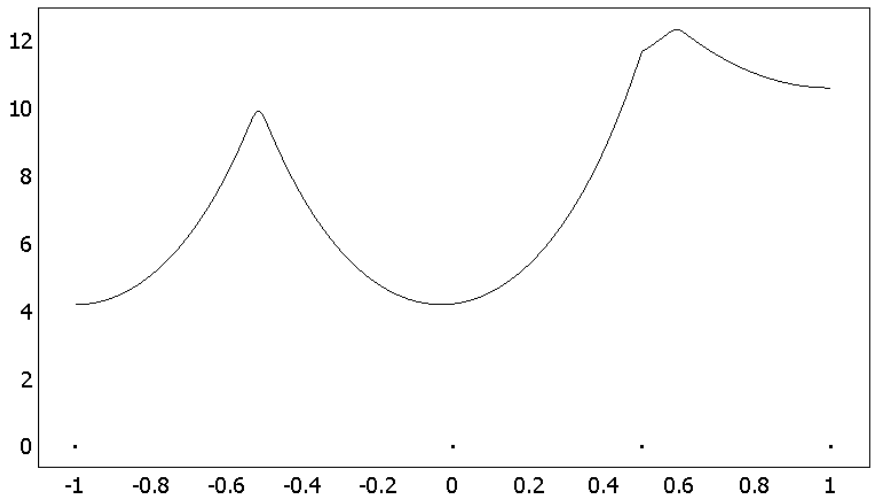

Figure 5. Long-time limit of the solution to (1.1) - (1.3) with $\epsilon^{2}=0.0001$ and $D(x)=0.1$ for $-1<x<0.5$, $D(x)=0.5$ for $0.5<x<1$. The initial conditions are given in Figure 1 . We observe an interior spike in the left subinterval combined with a spike near the jump discontinuity or two interior spikes in different subintervals. 
We conclude the computations by showing the results of some two-dimensional computations. The domain is a disc and the diffusion coefficient of the inhibitor jumps along a circle: it is smaller on a small inner disc and larger on an outer annulus. We observe multiple spikes which are denser and have smaller amplitude in the region with smaller diffusion coefficient.

In this figure, we display only the activator, $a$. We give a $2-D$ and a $3-D$ representation of the solution at $t=10^{5}$.
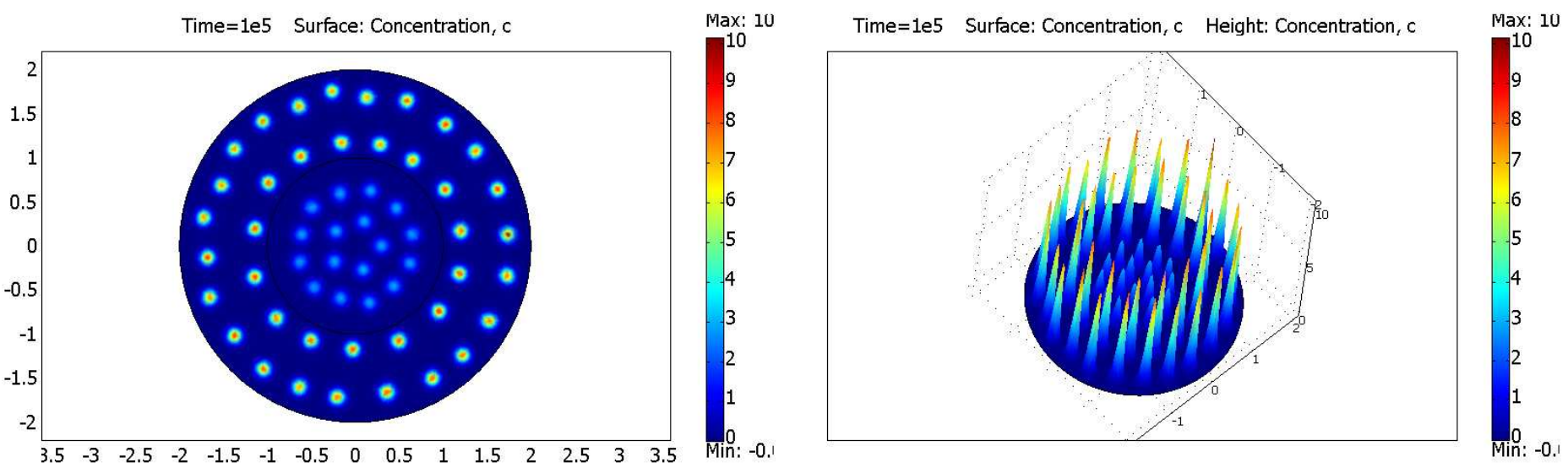

Figure 6. Long-time limit of the solution to (1.1) - (1.3) in the disc $|x|<2$, where $\epsilon^{2}=0.001$ and $D(x)=0.01$ for $|x|<1, D(x)=0.05$ for $1<|x|<2$. In the inner disc of the domain, where the diffusion coefficient is smaller than in the outer annulus, we observe multiple spikes which are denser and have smaller amplitude. 


\section{Discussion}

It is seen in the numerical computations given in the previous section that dynamically, if a spike moves from the left or right boundary, it converges to the closest stationary stable spike, either an interior spike or a spike near the jump discontinuity. This includes the possibility that the spike crosses the jump discontinuity and moves from one subinterval into the other if there is no stable spike at the jump discontinuity.

The numerical computations support the conjecture that one of spikes near the jump discontinuity given in Theorem 3.4 is stable while the other one is unstable. The dynamical system seems to select this stable position in the long-time limit. However, the spike does not stop at the unstable position.

This dynamical behavior is simpler than in [20]. For example, we do not observe rebounding or oscillatory spikes.

Steady states with two spikes (which are possible for smaller diffusion constants) display several interesting phenomena. Numerical computations show that many combinations are possible, such as two interior spikes in the same subinterval, two interior spikes in different subintervals or a spike near the jump discontinuity and an interior spike. We plan to analyze these interesting effects in future work. In these solutions the two spikes in general have different amplitudes unless they are both located in the same subinterval.

We now discuss the formation of boundaries resulting form varying genetic expressions. These results have been reviewed in [4], [5], [14].

The A-P and D-V boundaries in the Drosophila wing imaginal disc and the compartments in the vertebrate hindbrain are examples of lineage boundaries: they are established because of varying gene expressions in different compartments, and due to varying adhesion effects only a small percentage of cells cross the boundary to move into the neighboring compartment. The imaginal disc consists of a group of cells which form adult structures during metamorphosis. Investigating the mechanisms of their interaction is crucial in understanding how a larva develops into a fly. After establishment of the compartments often special border cells are created. They play an 
important role for morphogenesis by acting as a signaling center which determines the further progress of patterning.

We now discuss the genetic basis for the formation of a domain boundary in these examples:

- In the A-P Drosophila wing compartment border, the Hedgehog signaling pathway plays an important role by activating the secreted signaling molecule DPP, which acts as a long-range morphogen. DPP controls the further subdivision of the wing and formation of extra borders such as stripes of specialized cells or the wing veins [14].

- In the D-V Drosophila wing compartment border, the Notch signaling pathway plays a major role. It has been found that the following pathway molecules are important: Fringe stabilizes the Notch feedback loop at the border ([23], reviewed in [4]). Notch activates Wingless which acts as a long-range morphogen. It has recently been shown that the Myosin II molecule which is regulated downstream of Notch signaling accumulates at the border and that it is required to maintain the $\mathrm{D}-\mathrm{V}$ border $[16]$.

- The vertebrate hindbrain compartments are formed by signaling of Ephrin receptors which changes the adhesion properties in neighboring compartments (reviewed in [14]).

- The compartments of the zebrafish hindbrain are well-known lineage compartments. It has been shown ([7], reviewed in [5]) that boundary cells have migratory properties different from neighboring cells, a process which is driven by the Notch signaling pathway.

A model which explains the role of boundaries as organizing regions for secondary embryonic fields has been introduced by Meinhardt [18]. Within this framework, the primary orientation (head to tail) is determined by positional information [35]. Then, due to the fact that a high concentration of morphogen is present at the boundary, finer secondary structures are formed. Various biological examples can be explained using this model, such as the formation of duplicated and triplicated insect legs and the regeneration-duplication phenomenon of imaginal disc fragments.

The effects explored in this paper such as asymmetric positions or amplitudes of spikes as well as localization of patterns to a sub-domain play an important role in biological modeling, for example in the development of skeletal patterns in growing limbs or for compartment boundaries. We plan to 
shed further light on these issues in the future, combining analysis with computation and applying the outcomes to various biological models.

\section{Appendix A: introduction and analysis of the Green's FunCtion}

Let $G\left(x, t_{0}\right)$ be the Green's function which is defined as the unique solution of the problem

$$
\left\{\begin{aligned}
&\left(D(x) G\left(x, t_{0}\right)_{x}\right)_{x}-G\left(x, t_{0}\right)+\delta_{t_{0}}=0, G_{x}\left(-1, t_{0}\right)=G_{x}\left(1, t_{0}\right)=0, \\
& D\left(t_{0}\right) G_{x}\left(t_{0}^{-}, t_{0}\right)-D\left(t_{0}\right) G_{x}\left(t_{0}^{+}, t_{0}\right)=1, \quad G\left(t_{0}^{-}, t_{0}\right)-G\left(t_{0}^{+}, t_{0}\right)=0, \\
& D\left(x_{b}^{-}\right) G_{x}\left(x_{b}^{-}, t_{0}\right)-D\left(x_{b}^{+}\right) G_{x}\left(x_{b}^{+}, t_{0}\right)=0, G\left(x_{b}^{-}, t_{0}\right)-G\left(x_{b}^{+}, t_{0}\right)=0,
\end{aligned}\right.
$$

where $\delta_{t_{0}}$ is the Dirac delta distribution located at $t_{0}$.

Setting

$$
G\left(x, t_{0}\right)= \begin{cases}A \frac{\cosh \theta_{1}(x+1)}{\cosh \theta_{1}\left(t_{0}+1\right)}, & -1<x<t_{0}, \\ B \frac{\sinh \theta_{1}\left(x-t_{0}\right)}{\sinh \theta_{1}\left(x_{b}-t_{0}\right)}+A \frac{\sinh \theta_{1}\left(x-x_{b}\right)}{\sinh \theta_{1}\left(t_{0}-x_{b}\right)}, & t_{0}<x<x_{b}, \\ B \frac{\cosh \theta_{2}(x-1)}{\cosh \theta_{2}\left(x_{b}-1\right)}, & x_{b}<x<1\end{cases}
$$

then $G\left(x, t_{0}\right)$ is continuous at both $x=t_{0}$ and $x=x_{b}$. Using the conditions that $D(x) G_{x}\left(x, t_{0}\right)$ jumps by -1 at $x=t_{0}$ and is continuous at $x=x_{b}$, we get

$$
\begin{array}{r}
\frac{A}{\theta_{1}}\left(\tanh \theta_{1}\left(t_{0}+1\right)+\operatorname{coth} \theta_{1}\left(x_{b}-t_{0}\right)\right)-\frac{B}{\theta_{1}} \frac{1}{\sinh \theta_{1}\left(x_{b}-t_{0}\right)}=1, \\
B\left(\frac{1}{\theta_{1}} \operatorname{coth} \theta_{1}\left(x_{b}-t_{0}\right)+\frac{1}{\theta_{2}} \tanh \theta_{2}\left(1-x_{b}\right)\right)-\frac{A}{\theta_{1}} \frac{1}{\sinh \theta_{1}\left(x_{b}-t_{0}\right)}=0 .
\end{array}
$$

From (9.3) we compute

$$
\begin{aligned}
& G\left(t_{0}, t_{0}\right)^{-1}=A^{-1}=\theta_{1}^{-1}\left[\tanh \theta_{1}\left(t_{0}+1\right)+\operatorname{coth} \theta_{1}\left(x_{b}-t_{0}\right)\right. \\
& \left.-\left(\sinh \theta_{1}\left(x_{b}-t_{0}\right) \cosh \theta_{1}\left(x_{b}-t_{0}\right)+\frac{\theta_{1}}{\theta_{2}} \sinh ^{2} \theta_{1}\left(x_{b}-t_{0}\right) \tanh \theta_{2}\left(1-x_{b}\right)\right)^{-1}\right] \\
& =\theta_{1}^{-1}\left[\tanh \theta_{1}\left(t_{0}+1\right)+\frac{\theta_{2} \sinh \theta_{1}\left(x_{b}-t_{0}\right)+\theta_{1} \cosh \theta_{1}\left(x_{b}-t_{0}\right) \tanh \theta_{2}\left(1-x_{b}\right)}{\theta_{2} \cosh \theta_{1}\left(x_{b}-t_{0}\right)+\theta_{1} \sinh \theta_{1}\left(x_{b}-t_{0}\right) \tanh \theta_{2}\left(1-x_{b}\right)}\right]=: \theta_{1}^{-1} u\left(t_{0}\right) .
\end{aligned}
$$

Setting $v\left(t_{0}\right)=\theta_{2} \cosh \theta_{1}\left(x_{b}-t_{0}\right)+\theta_{1} \sinh \theta_{1}\left(x_{b}-t_{0}\right) \tanh \theta_{2}\left(1-x_{b}\right)$, we have

$$
u\left(t_{0}\right)=\tanh \theta_{1}\left(t_{0}+1\right)-\theta_{1}^{-1} \frac{v^{\prime}\left(t_{0}\right)}{v\left(t_{0}\right)} .
$$


Note that $\theta_{1}^{-2} v^{\prime \prime}\left(t_{0}\right)=v\left(t_{0}\right)$. This implies for $u^{\prime}\left(t_{0}\right)=\frac{d}{d t_{0}} u\left(t_{0}\right)$ that

$$
\begin{aligned}
\theta_{1}^{-1} u^{\prime}\left(t_{0}\right) & =1-\tanh ^{2} \theta_{1}\left(t_{0}+1\right)-\theta_{1}^{-2} \frac{v^{\prime \prime}\left(t_{0}\right) v\left(t_{0}\right)-\left(v^{\prime}\left(t_{0}\right)\right)^{2}}{\left(v\left(t_{0}\right)\right)^{2}} \\
& =-\tanh ^{2} \theta_{1}\left(t_{0}+1\right)+\theta_{1}^{-2} \frac{\left(v^{\prime}\left(t_{0}\right)\right)^{2}}{\left(v\left(t_{0}\right)\right)^{2}} .
\end{aligned}
$$

Note that $\frac{d}{d t_{0}} G\left(t_{0}, t_{0}\right)=0$ iff $u^{\prime}\left(t_{0}\right)=0$ and $u\left(t_{0}\right) \neq 0$, since

$$
\frac{d}{d t_{0}} G\left(t_{0}, t_{0}\right)=-\theta_{1} \frac{u^{\prime}\left(t_{0}\right)}{\left(u\left(t_{0}\right)\right)^{2}}
$$

Next we compute

$$
\begin{aligned}
\theta_{1}^{-2} u^{\prime \prime}\left(t_{0}\right)= & -2 \tanh \theta_{1}\left(t_{0}+1\right)\left(1-\tanh ^{2} \theta_{1}\left(t_{0}+1\right)\right) \\
& +2 \theta_{1}^{-3} \frac{v\left(t_{0}\right) v^{\prime}\left(t_{0}\right) v^{\prime \prime}\left(t_{0}\right)-v\left(t_{0}\right)\left(v^{\prime}\left(t_{0}\right)\right)^{3}}{\left(v\left(t_{0}\right)\right)^{3}} \\
= & -2 \tanh \theta_{1}\left(t_{0}+1\right)\left(1-\tanh ^{2} \theta_{1}\left(t_{0}+1\right)\right) \\
& +2 \theta_{1}^{-3} \frac{v^{\prime}\left(t_{0}\right)\left[\theta_{1}^{2}\left(v\left(t_{0}\right)\right)^{2}-\left(v^{\prime}\left(t_{0}\right)\right)^{2}\right]}{\left(v\left(t_{0}\right)\right)^{3}}
\end{aligned}
$$

Using the relations

$$
\theta_{1}^{2}\left(v\left(t_{0}\right)\right)^{2}-\left(v^{\prime}\left(t_{0}\right)\right)^{2}=\theta_{1}^{2} \theta_{2}^{2}\left[1-\left(\frac{\theta_{1}}{\theta_{2}} \tanh \theta_{2}\left(1-x_{b}\right)\right)^{2}\right]>0
$$

(by (3.1)) and $v^{\prime}\left(t_{0}\right)<0$, we get $u^{\prime \prime}\left(t_{0}\right)<0$. To determine the sign of $\frac{d^{2}}{d t_{0}^{2}} G\left(t_{0}, t_{0}\right)$, we compute

$$
\frac{d^{2}}{d t_{0}^{2}} G\left(t_{0}, t_{0}\right)=\theta_{1} \frac{-u^{\prime \prime}\left(t_{0}\right) u\left(t_{0}\right)+2\left(u^{\prime}\left(t_{0}\right)\right)^{2}}{\left(u\left(t_{0}\right)\right)^{3}} .
$$

Noting that $u^{\prime}\left(t_{0}\right)=0$ and $u^{\prime \prime}\left(t_{0}\right)<0$, we get

$$
\frac{d^{2}}{d t_{0}^{2}} G\left(t_{0}, t_{0}\right)>0
$$

This will imply that the interior spike is linearly stable. The proof of this statement will be given in Appendix E.

Now we study further properties of the Green's function $G$. For $t_{0} \in(-1,1)$ we consider $G\left(t_{0}, t_{0}\right)$. We define the regular part of the Green's function as

$$
H(x, y):=\frac{\theta_{i}}{2} e^{-\theta_{i}|x-y|}-G(x, y)
$$

Then we compute

$$
\frac{d}{d t_{0}} G\left(t_{0}, t_{0}\right)=\frac{d}{d t_{0}} \frac{\theta_{i}}{2}-\frac{d}{d t_{0}} H\left(t_{0}, t_{0}\right)=-\left.2 \nabla_{x}\right|_{x=t_{0}} H\left(x, t_{0}\right)=:-2 \nabla_{t_{0}} H\left(t_{0}, t_{0}\right),
$$


where $i=1$ if $t_{0}<x_{b}$ and $i=2$ if $t_{0}>x_{b}$. Here we have used the symmetry of $H(x, y)$ and the notation

$$
\left.\nabla_{x}\right|_{x=t_{0}} H\left(x, t_{0}\right):=\left.\frac{\partial}{\partial x}\right|_{x=t_{0}} H\left(x, t_{0}\right) .
$$

Further, we compute

$$
\frac{d^{2}}{d t_{0}^{2}} G\left(t_{0}, t_{0}\right)=-2\left(\nabla_{x} \nabla_{y}\right)_{x=y=t_{0}} H(x, y)-2\left(\nabla_{x}^{2}\right)_{x=t_{0}} H\left(x, t_{0}\right)=:-2 \nabla_{t_{0}}^{2} H\left(t_{0}, t_{0}\right) .
$$

Note that $\hat{\xi}(t)$, which has been defined in (4.6), is in $C^{1}(-1,1)$. We now compute $\nabla_{t} \hat{\xi}(t)$ :

$$
\nabla_{t} \hat{\xi}(t)=\frac{d}{d t}(G(t, t))^{-1}=2 \nabla_{t}(G(t, t))^{-1}=-2\left(\nabla_{t} G(t, t)\right) \hat{\xi}(t)^{2} .
$$

We also need to know the derivative of the function

$$
F(t):=\left(-2 \nabla_{t} G(t, t)\right) \hat{\xi}^{2}(t)=\nabla_{t} \hat{\xi}(t)
$$

We compute

$$
\nabla_{t} F(t)=\nabla_{t} \frac{-2 \nabla_{t} G(t, t)}{G^{2}(t, t)}=\frac{-2 G(t, t) \nabla_{t}^{2} G(t, t)+4\left(\nabla_{t} G(t, t)\right)^{2}}{G^{3}(t, t)},
$$

which implies that

$$
\nabla_{t_{0}} F\left(t_{0}\right)=\frac{-2 G\left(t_{0}, t_{0}\right) \nabla_{t_{0}}^{2} G\left(t_{0}, t_{0}\right)}{G^{3}\left(t_{0}, t_{0}\right)}
$$

if $\nabla_{t_{0}} G\left(t_{0}, t_{0}\right)=0$ which will be assumed in (12.7) below.

\section{Appendix B: estimates for the approximate steady state}

For $A=w_{\epsilon, t}$, where $w_{\epsilon, t}$ is defined in (4.9), let us compute

$$
\tau:=T[A]\left(t_{0}\right)
$$

From (4.10), we have

$$
\begin{aligned}
\tau & =T[A]\left(t_{0}\right)=\int_{-1}^{1} G\left(t_{0}, z\right) A^{2}(z) d z \\
& =\xi_{0}^{2} \int_{-1}^{1} G\left(t_{0}, z\right) \tilde{w}_{0}^{2}(z) d z \\
& =\xi_{0}^{2} \epsilon\left[G\left(t_{0}, t_{0}\right) \int_{-\infty}^{+\infty} w^{2}(y) d y+O(\epsilon)\right] \\
& =\xi_{\epsilon}\left[G\left(t_{0}, t_{0}\right) \hat{\xi}_{0}^{2}+O(\epsilon)\right] \quad(\text { by }(4.8)) \\
& =\xi_{\epsilon}\left[\hat{\xi}_{0}+O(\epsilon)\right] \quad(\text { by }(4.6)) .
\end{aligned}
$$


Let $x=t_{0}+\epsilon y, \mathbf{z}=\mathbf{t}_{\mathbf{0}}+\epsilon \tilde{\mathbf{z}}$, where $\mathbf{x}, \mathbf{z} \in \mathbf{B}_{\epsilon^{3 / 4}}\left(\mathbf{t}_{\mathbf{0}}\right)$. We calculate

$$
\begin{aligned}
& T[A](x)-T[A]\left(t_{0}\right)= \\
&=\int_{-1}^{1}\left[G(x, z)-G\left(t_{0}, z\right)\right] A^{2}(z) d z \\
&=\xi_{0}^{2} \int_{-1}^{1}\left[G(x, z)-G\left(t_{0}, z\right)\right] \tilde{w}_{0}^{2}(z) d z \\
&=\xi_{0}^{2} \int_{-1}^{1}\left[K(|x-z|)-K\left(\left|t_{0}-z\right|\right)\right] \tilde{w}_{0}^{2}(z) d z-\xi_{0}^{2} \int_{-1}^{1}\left[H(x, z)-H\left(t_{0}, z\right)\right] \tilde{w}_{0}^{2}(z) d z \\
&=\epsilon^{2} \xi_{0}^{2}\left[\int_{-\infty}^{+\infty}\left[\frac{\theta_{\mathbf{i}}^{2}}{\mathbf{2}}|\tilde{z}|-\frac{\theta_{\mathbf{i}}^{2}}{\mathbf{2}}|y-\tilde{z}|\right] w^{2}(\tilde{z}) d \tilde{z}+O\left(\epsilon y^{2}+\epsilon^{2}\right)\right] \\
& \quad+\epsilon^{2} \xi_{0}^{2}\left[-\left.\nabla_{x} H\left(x, t_{0}\right)\right|_{x=t_{0}} y \int_{-\infty}^{+\infty} w^{2}(z) d z+O\left(\epsilon y^{2}+\epsilon^{2}\right)\right] \\
&=\epsilon^{2} \xi_{0}^{2} P_{0}(y)+\epsilon^{2} \xi_{0}^{2} \int_{-\infty}^{+\infty} w^{2}(z) d z\left[-\left.\nabla_{x} H\left(x, t_{0}\right)\right|_{x=t_{0}}\right] y+O\left(\epsilon y^{2}+\epsilon^{2}\right) \\
&= \epsilon \xi_{\epsilon}\left\{\hat{\xi}_{0}^{2} \frac{P_{0}(y)}{\int_{-\infty}^{\infty} w^{2}(z) d z}+\hat{\xi}_{0}^{2}\left[-\left.\nabla_{x} H\left(x, t_{0}\right)\right|_{x=t_{0}}\right] y+O\left(\epsilon y^{2}+\epsilon^{2}\right)\right\}
\end{aligned}
$$

by (4.8), where

$$
P_{0}(y)=\int_{-\infty}^{+\infty}\left[\frac{\theta_{\mathbf{i}}^{2}}{\mathbf{2}}|z|-\frac{\theta_{\mathbf{i}}^{2}}{\mathbf{2}}|y-z|\right] w^{2}(z) d z
$$

Note that the function $P_{0}(y)$ is even in $y$.

For a function $A \in L^{2}(-1,1)$, let

$$
S_{\epsilon}[A]=\epsilon^{2} \Delta A-A+\frac{A^{2}}{T[A]},
$$

where $T[A]$ is given by (4.10). We now set $A=w_{\epsilon, t}$ and compute $S_{\epsilon}\left[w_{\epsilon, t}\right]$. In fact,

$$
\begin{aligned}
S_{\epsilon}\left[w_{\epsilon, t}\right] & =\epsilon^{2} \Delta w_{\epsilon, t}-w_{\epsilon, t}+\frac{w_{\epsilon, t}^{2}}{T\left[w_{\epsilon, t}\right]} \\
& =\xi_{0}\left(\epsilon^{2} \Delta \tilde{w}_{0}-\tilde{w}_{0}\right)+\frac{w_{\epsilon, t}^{2}}{T\left[w_{\epsilon, t}\right]}+\text { e.s.t. } \\
& =\frac{\xi_{0}^{2} \tilde{w}_{0}^{2}}{T\left[w_{\epsilon, t}\right]}-\xi_{0} \tilde{w}_{0}^{2}+\text { e.s.t. } \\
& =E_{1}+\text { e.s.t. }
\end{aligned}
$$

where

$$
E_{1}=\frac{\xi_{0}^{2} \tilde{w}_{0}^{2}}{T\left[w_{\epsilon, t}\right]}-\xi_{0} \tilde{w}_{0}^{2}
$$


Now we estimate $E_{1}$, using (10.2), (10.3):

$$
\begin{aligned}
& \xi_{\epsilon}^{-1} E_{1}=\frac{\xi_{0}^{2} \tilde{w}_{0}^{2}}{T\left[w_{\epsilon, t}\right]} \xi_{\epsilon}^{-1}-\hat{\xi}_{0} \tilde{w}_{0}^{2} \\
& =\frac{\left(\xi_{0} \tilde{w}_{0}\right)^{2}}{T\left[w_{\epsilon, t}\right]\left(t_{0}\right)} \xi_{\epsilon}^{-1}-\hat{\xi}_{0} \tilde{w}_{0}^{2}-\frac{\left(\xi_{0} \tilde{w}_{0}\right)^{2}}{\left(T\left[w_{\epsilon, t}\right]\left(t_{0}\right)\right)^{2}}\left(T\left[w_{\epsilon, t}\right]-T\left[w_{\epsilon, t}\right]\left(t_{0}\right)\right) \xi_{\epsilon}^{-1} \\
& +O\left(\left|T\left[w_{\epsilon, t}\right]-T\left[w_{\epsilon, t}\right]\left(t_{0}\right)\right|^{2} \epsilon \tilde{w}_{0}^{2}\right) \\
& =\tilde{w}_{0}^{2}\left(\frac{\hat{\xi}_{0}^{2}}{\hat{\xi}_{0}}-\hat{\xi}_{0}\right)-\hat{\xi}_{0} \tilde{w}_{0}^{2} \frac{T\left[w_{\epsilon, t}\right]-T\left[w_{\epsilon, t}\right]\left(t_{0}\right)}{T\left[w_{\epsilon, t}\right]\left(t_{0}\right)}+O\left(\epsilon^{2} y^{2} \tilde{w}_{0}^{2}\right) \\
& =-\epsilon \tilde{w}_{0}^{2}\left\{\hat{\xi}_{0}^{2} \frac{P_{0}(\mathbf{y})}{\int_{-\infty}^{\infty} w^{2}(z) d z}+\hat{\xi}_{0}^{2}\left[-\left.\nabla_{x} H\left(x, t_{0}\right)\right|_{x=t_{0}}\right] \mathbf{y}\right\}+O\left(\epsilon^{2} y^{2} \tilde{w}_{0}^{2}\right) \text {. }
\end{aligned}
$$

This implies that

$$
\xi_{\epsilon}^{-1}\left\|E_{1}\right\|_{L^{2}(R)}=O(\epsilon)
$$

From (10.6), we conclude that

$$
\xi_{\epsilon}^{-1}\left\|S_{\epsilon}\left[\mathbf{w}_{\epsilon, \mathbf{t}}\right]\right\|_{L^{2}(R)}=O(\epsilon)
$$

\section{Appendix C: the Liapunov-Schmidt Reduction}

In this appendix we study the linear operator defined by

$$
\tilde{L}_{\epsilon, t} \phi:=S_{\epsilon}^{\prime}[A] \phi=\epsilon^{2} \Delta \phi-\phi+\frac{2 A \phi}{T[A]}-\frac{A^{2}}{(T[A])^{2}}\left(T^{\prime}[A] \phi\right), \quad H_{N}^{2}(\Omega) \rightarrow L^{2}(\Omega),
$$

where $A=w_{\epsilon, t}$ and for $\phi \in L^{2}(\Omega)$ the function $T^{\prime}[A] \phi$ is defined as the unique solution in $H_{N}^{2, *}(\Omega)$ of

$$
\left(D(x)\left(T^{\prime}[A] \phi\right)_{x}\right)_{x}-\left(T^{\prime}[A] \phi\right)+2 A \phi=0, \quad-1<x<1 .
$$

We define the approximate kernel and co-kernel of the operator $\tilde{L}_{\epsilon, t}$ as follows:

$$
\begin{aligned}
& \mathcal{K}_{\epsilon, t}:=\operatorname{span}\left\{\frac{d \tilde{w}_{0}}{d x}\right\} \subset H_{N}^{2}(\Omega), \\
& \mathcal{C}_{\epsilon, \mathbf{t}}:=\operatorname{span}\left\{\frac{d \tilde{w}_{0}}{d x}\right\} \subset L^{2}(\Omega) .
\end{aligned}
$$

We also introduce the orthogonal projection $\pi_{\epsilon, t}^{\perp}: L^{2}(\Omega) \rightarrow \mathcal{C}_{\epsilon, t}^{\perp}$ and study the operator $L_{\epsilon, t}:=\pi_{\epsilon, t}^{\perp} \circ \tilde{L}_{\epsilon, t}$. By letting $\epsilon \rightarrow 0$, we will show that $L_{\epsilon, t}: \mathcal{K}_{\epsilon, t}^{\perp} \rightarrow \mathcal{C}_{\epsilon, t}^{\perp}$ is invertible with a uniformly bounded inverse provided $\epsilon$ is sufficiently small. This statement is contained in the following proposition. 
Proposition 11.1. There exist positive constants $\bar{\epsilon}, \lambda$ such that for all $\epsilon \in(0, \bar{\epsilon})$ and all $t \in B_{\epsilon^{3 / 4}}\left(t_{0}\right)$ we have

$$
\left\|L_{\epsilon, t} \phi_{\epsilon}\right\|_{L^{2}\left(\Omega_{\epsilon}\right)} \geq \lambda\left\|\phi_{\epsilon}\right\|_{H^{2}\left(\Omega_{\epsilon}\right)}
$$

Further, the map

$$
L_{\epsilon, t}=\pi_{\epsilon, t}^{\perp} \circ \tilde{L}_{\epsilon, t}: \mathcal{K}_{\epsilon, t}^{\perp} \rightarrow \mathcal{C}_{\epsilon, t}^{\perp}
$$

is surjective.

Proof: The proof is given in Proposition 5.1 of [34].

Now we are in a position to solve the equation

$$
\pi_{\epsilon, t}^{\perp} \circ S_{\epsilon}\left(w_{\epsilon, t}+\phi\right)=0, \quad \phi \in \mathcal{K}_{\epsilon, \mathbf{t}}^{\perp}
$$

Since by Proposition 11.1 $L_{\epsilon, t}: \mathcal{K}_{\epsilon, t}^{\perp} \rightarrow \mathcal{C}_{\epsilon, t}^{\perp}$ is invertible (call the inverse $L_{\epsilon, t}^{-1}$ ), this is equivalent to

$$
\phi=-\left(L_{\epsilon, t}^{-1} \circ \pi_{\epsilon, t}^{\perp}\right)\left(S_{\epsilon}\left[w_{\epsilon, t}\right]\right)-\left(L_{\epsilon, t}^{-1} \circ \pi_{\epsilon, t}^{\perp}\right)\left(N_{\epsilon, t}[\phi]\right)=: M_{\epsilon, t}[\phi], \quad \phi \in \mathcal{K}_{\epsilon, \mathbf{t}}^{\perp},
$$

where

$$
N_{\epsilon, t}[\phi]=S_{\epsilon}\left[w_{\epsilon, t}+\phi\right]-S_{\epsilon}\left[w_{\epsilon, t}\right]-S_{\epsilon}^{\prime}\left[w_{\epsilon, t}\right] \phi
$$

We are going to show that the operator $M_{\epsilon, t}$ defined by (11.4) is a contraction mapping on

$$
\mathbf{B}_{\epsilon, \delta}:=\left\{\phi \in \mathcal{K}_{\epsilon, \mathbf{t}}^{\perp}:\|\phi\|_{\mathbf{H}^{2}\left(\Omega_{\epsilon}\right)}<\delta\right\}
$$

if $\delta$ and $\epsilon$ are sufficiently small. We have by (10.9) and Proposition 11.1

$$
\begin{aligned}
\xi_{\epsilon}^{-1}\left\|M_{\epsilon, t}[\phi]\right\|_{H^{2}\left(\Omega_{\epsilon}\right)} & \leq \lambda^{-1} \xi_{\epsilon}^{-1}\left(\left\|\pi_{\epsilon, t}^{\perp}\left(N_{\epsilon, t}[\phi]\right)\right\|_{L^{2}\left(\Omega_{\epsilon}\right)}+\left\|\pi_{\epsilon, t}^{\perp}\left(S_{\epsilon}\left[w_{\epsilon, t}\right]\right)\right\|_{L^{2}\left(\Omega_{\epsilon}\right)}\right) \\
& \leq \lambda^{-1} C\left(\xi_{\epsilon}^{-1} \delta^{2}+\epsilon\left|\nabla_{t} G(t, t)\right|\right)
\end{aligned}
$$

where $\lambda>0, \mathbf{C}>\mathbf{0}$ are independent of $\delta>0, \epsilon>0$. Similarly, it follows that

$$
\xi_{\epsilon}^{-1}\left\|M_{\epsilon, t}[\phi]-M_{\epsilon, t}\left[\phi^{\prime}\right]\right\|_{H^{2}\left(\Omega_{\epsilon}\right)} \leq \lambda^{-1} C\left(\xi_{\epsilon}^{-1} \delta^{2}+\epsilon\left|\nabla_{t} G(t, t)\right|\right)\left\|\phi-\phi^{\prime}\right\|_{H^{2}\left(\Omega_{\epsilon}\right)}
$$

where $\lambda>0, \mathbf{C}>\mathbf{0}$ are independent of $\delta>0, \epsilon>0$. 
By the previous two estimates, if we choose $\delta$ and $\epsilon$ sufficiently small, then $M_{\epsilon, t}$ is a contraction mapping on $B_{\epsilon, \delta}$. The existence of a fixed point $\phi_{\epsilon, t}$ now follows from the contraction mapping principle and $\phi_{\epsilon, t}$ is the unique solution of (11.4).

We have thus proved the following result.

Lemma 11.2. There exists $\bar{\epsilon}>0$ such that for every pair of $\epsilon, t$ with $0<\epsilon<\bar{\epsilon}$ and $t \in B_{\epsilon^{3 / 4}}\left(t_{0}\right)$ there exists a unique $\phi_{\epsilon, t} \in \mathcal{K}_{\epsilon, \mathbf{t}}^{\perp}$ satisfying $S_{\epsilon}\left(w_{\epsilon, t}+\phi_{\epsilon, t}\right) \in \mathcal{C}_{\epsilon, t}$. Further, we have the estimate

$$
\xi_{\epsilon}^{-1}\left\|\phi_{\epsilon, t}\right\|_{H^{2}\left(\Omega_{\epsilon}\right)} \leq C \epsilon
$$

\section{Appendix D: THE REDUCED PROBleM}

In this appendix we derive a reduced problem which will be essential for the proof of the existence results.

By Lemma 11.2, for every $t \in B_{\epsilon^{3 / 4}}\left(t_{0}\right)$ there exists a unique solution $\phi_{\epsilon, t} \in \mathcal{K}_{\epsilon, t}^{\perp}$ such that

$$
S_{\epsilon}\left[w_{\epsilon, t}+\phi_{\epsilon, t}\right]=v_{\epsilon, t} \in \mathcal{C}_{\epsilon, t}
$$

Our idea is to find $t^{\epsilon} \in B_{\epsilon^{3 / 4}}\left(t_{0}\right)$ such that in addition

$$
S_{\epsilon}\left[w_{\epsilon, t^{\epsilon}}+\phi_{\epsilon, t^{\epsilon}}\right] \perp \mathcal{C}_{\epsilon, t^{\epsilon}}
$$

Then from (12.1) and (12.2) we get that $S_{\epsilon}\left[w_{\epsilon, t^{\epsilon}}+\phi_{\epsilon, t^{\epsilon}}\right]=0$. To this end, we let

$$
W_{\epsilon}(t):=\xi_{\epsilon}^{-1} \epsilon^{-1} \int_{\Omega} S\left[w_{\epsilon, t}+\phi_{\epsilon, t}\right] \frac{d \tilde{w}_{0}}{d x} d x, \quad B_{\epsilon^{3 / 4}}\left(t_{0}\right) \rightarrow R .
$$

Then the problem is reduced to finding a zero of the function $W_{\epsilon}(t)$ in $\mathbf{B}_{\epsilon^{3 / 4}}\left(\mathbf{t}_{\mathbf{0}}\right)$.

Let us now calculate $W_{\epsilon}(t)$. We have

$$
\begin{aligned}
\xi_{\epsilon}^{-1} \epsilon^{-1} \int_{\Omega} S\left[w_{\epsilon, t}+\phi_{\epsilon, t}\right] \frac{d \tilde{w}_{0}}{d x} d x= & \xi_{\epsilon}^{-1} \epsilon^{-1} \int_{\Omega} S\left[w_{\epsilon, t}\right] \frac{d \tilde{w}_{0}}{d x} d x \\
& +\xi_{\epsilon}^{-1} \epsilon^{-1} \int_{\Omega} S_{\epsilon}^{\prime}\left[w_{\epsilon, t}\right] \phi_{\epsilon, t} \frac{d \tilde{w}_{0}}{d x} d x+\xi_{\epsilon}^{-1} \epsilon^{-1} \int_{\Omega} N_{\epsilon, t}\left[\phi_{\epsilon, t}\right] \frac{d \tilde{w}_{0}}{d x} d x \\
= & I_{1}+I_{2}+I_{3},
\end{aligned}
$$

where $I_{1}, I_{2}$ and $I_{3}$ are defined by the last equality. 
The computation of $I_{3}$ is the easiest: note that the first term in the expansion of $N_{\epsilon}$ is quadratic in $\phi_{\epsilon, t}$ and so

$$
I_{3}=O\left(\epsilon^{2}\right)
$$

Now we compute $I_{1}$ and $I_{2}$. The result will be that $I_{1}$ is the leading term and $I_{2}=O(\epsilon)$.

For $I_{1}$, we have

$$
I_{1}=\xi_{\epsilon}^{-1} \epsilon^{-1} \int_{\Omega} E_{1} \frac{d \tilde{w}_{0}}{d x} d x+O(\epsilon)
$$

where $E_{1}$ has been defined in (10.7).

We calculate, using $(10.8)$ and the fact that $P_{0}(y)$ is an even function

$$
\begin{aligned}
I_{1} & =\xi_{\epsilon}^{-1} \epsilon^{-1} \int_{\Omega} E_{1} \frac{d \tilde{w}_{0}}{d x} d x \\
& =-\int_{R} \tilde{w}_{0}^{2}\left[\hat{\xi}_{0}^{2}\left[-\left.\nabla_{x} H\left(x, t_{0}\right)\right|_{x=t_{0}}\right] y\right] \tilde{w}_{0}^{\prime} d y+O(\epsilon) \\
& =\hat{\xi}_{0}^{2} \int_{R}\left(y w^{2} w^{\prime}\right) d y\left[\left.\nabla_{x} H\left(x, t_{0}\right)\right|_{x=t_{0}}\right]+O(\epsilon) \\
& =-\frac{1}{3} \hat{\xi}_{0}^{2} \int_{R} w^{3} d y\left[\left.\nabla_{x} H\left(x, t_{0}\right)\right|_{x=t_{0}}\right]+O(\epsilon) .
\end{aligned}
$$

Using (2.3), we have

$$
I_{1}=-d_{00}\left[\left.\nabla_{\mathbf{x}} \mathbf{H}\left(\mathbf{x}, \mathbf{t}_{\mathbf{0}}\right)\right|_{\mathbf{x}=\mathbf{t}_{\mathbf{0}}}\right]+O(\epsilon)
$$

where

$$
d_{00}=2.4 \hat{\xi}_{0}^{2}
$$

For $I_{2}$, we calculate

$$
\begin{aligned}
I_{2}= & \xi_{\epsilon}^{-1} \epsilon^{-1} \int_{\Omega} S_{\epsilon}^{\prime}\left[w_{\epsilon, t}\right]\left(\phi_{\epsilon, t}\right) \frac{d \tilde{w}_{0}}{d x} d x \\
= & \xi_{\epsilon}^{-1} \epsilon^{-1} \int_{\Omega}\left[\epsilon^{2} \Delta \phi_{\epsilon, t}-\phi_{\epsilon, t}+\frac{2 w_{\epsilon, t} \phi_{\epsilon, t}}{T\left[w_{\epsilon, t}\right]}-\frac{w_{\epsilon, t}^{2}}{\left(T\left[w_{\epsilon, t}\right]\right)^{2}}\left(T^{\prime}\left[w_{\epsilon, t}\right] \phi_{\epsilon, t}\right)\right] \frac{d \tilde{w}_{0}}{d x} d x \\
= & \xi_{\epsilon}^{-1} \epsilon^{-1} \int_{\Omega}\left[\epsilon^{2} \Delta \frac{d \tilde{w}_{0}}{d x}-\frac{d \tilde{w}_{0}}{d x}+\frac{2 w_{\epsilon, t}}{T\left[w_{\epsilon, t}\right]\left(t_{0}\right)}\right] \phi_{\epsilon, t} d x \\
& +\xi_{\epsilon}^{-1} \epsilon^{-1} \int_{\Omega} \frac{2 w_{\epsilon, t}}{T\left[w_{\epsilon, t}\right]\left(t_{0}\right)} \phi_{\epsilon, t}\left(\frac{T\left[w_{\epsilon, t}\right]\left(t_{0}\right)-T\left[w_{\epsilon, t}\right]}{T\left[w_{\epsilon, t}\right]}\right) d x \\
& -\xi_{\epsilon}^{-1} \epsilon^{-1} \int_{\Omega} \frac{w_{\epsilon, t}^{2}}{\left(T\left[w_{\epsilon, t}\right]\right)^{2}}\left(T^{\prime}\left[w_{\epsilon, t}\right] \phi_{\epsilon, t}\right) \frac{d \tilde{w}_{0}}{d x} d x+O\left(\epsilon^{2}\right)=O(\epsilon)
\end{aligned}
$$


by (4.5), (10.2), (10.3) since

$$
\left\|\phi_{\epsilon, t}\right\|_{H^{2}\left(\Omega_{\epsilon}\right)}=O(\epsilon)
$$

Combining $I_{1}, I_{2}$ and $I_{3}$, we have

$$
W_{\epsilon}(t)=-d_{00}\left[\left.\nabla_{\mathbf{x}} \mathbf{H}\left(\mathbf{x}, \mathbf{t}_{\mathbf{0}}\right)\right|_{\mathbf{x}=\mathbf{t}_{\mathbf{0}}}\right]+O(\epsilon)
$$

where

$$
d_{00}=2.4 \hat{\xi}_{0}^{2}
$$

Let us for the moment assume that

$$
\nabla_{t_{0}} H\left(t_{0}, t_{0}\right)=0 \quad \text { and } \quad \nabla_{t_{0}}^{2} H\left(t_{0}\right) \neq 0
$$

We will check the conditions in (12.7) at the end of this appendix.

Then the function $W_{\epsilon}(t)$ satisfies $W_{\epsilon}(t)=-\mathbf{c} \nabla_{t_{0}}^{2} H\left(t_{0}, t_{0}\right)\left(t-t_{0}\right)+O(\epsilon)$ for some $c>0$.

Thus for $\epsilon$ small enough $\nabla_{t} H(t, t)$ has exactly one zero in $B_{\epsilon^{3 / 4}}\left(t_{0}\right)$. Further, it has opposite signs for the endpoints of $B_{\epsilon^{3 / 4}}\left(t_{0}\right)$. Since this property is also true for $W_{\epsilon}(t)$, by the intermediate value theorem it follows that, for $\epsilon$ small enough, there exists a $t^{\epsilon} \in B_{\epsilon^{3 / 4}}\left(t_{0}\right)$ such that $W_{\epsilon}\left(t^{\epsilon}\right)=0$ and $t_{\epsilon} \rightarrow t_{0}$ as $\epsilon \rightarrow 0$

Thus we have proved the following proposition.

Proposition 12.1. For $\epsilon$ sufficiently small there exists a point $t^{\epsilon} \in B_{\epsilon^{3 / 4}}\left(t_{0}\right)$ with $t_{\epsilon} \rightarrow t_{0}$ such that $W_{\epsilon}\left(t^{\epsilon}\right)=0$.

Finally, we prove Theorem 3.1.

Proof of Theorem 3.1: We mention the main steps. By Proposition 12.1, there exists a $t^{\epsilon} \in B_{\epsilon^{3 / 4}}\left(t_{0}\right)$ such that $t^{\epsilon} \rightarrow t_{0}$ and $W_{\epsilon}\left(t^{\epsilon}\right)=0$, or, expressed differently, $S_{\epsilon}\left[w_{\epsilon, t^{\epsilon}}+\phi_{\epsilon, t^{\epsilon}}\right]=0$. Let $w_{\epsilon}=w_{\epsilon, t^{\epsilon}}+\phi_{\epsilon, t^{\epsilon}}$. By the Maximum Principle, $w_{\epsilon}>0$. Moreover, by its construction, $w_{\epsilon}$ has all the properties required in Theorem 3.1. The proof is finished.

It remains to check the conditions in (12.7). We assume that

$$
-1<t_{0}<x_{b}<1
$$


(This condition holds without loss of generality. The case $-1<x_{b}<t_{0}<1$ follows by reflection about $x=0$ which reverses the signs of of $x_{b}$ and $t_{0}$ and swaps the two diffusion constants $\theta_{1}$ and $\theta_{2}$.)

By (9.5), (9.6) together with (9.7), (9.8) and (9.9), we see that $\nabla_{t_{0}} H\left(t_{0}, t_{0}\right)=0$ and $\nabla_{t_{0}}^{2} H\left(t_{0}, t_{0}\right) \neq 0$ if and only if $t_{0}$ is a solution of the equation

$$
\frac{\sinh \theta_{1}\left(t_{0}+1\right)}{\cosh \theta_{1}\left(t_{0}+1\right)}=\frac{\theta_{2} \sinh \theta_{1}\left(x_{b}-t_{0}\right)+\theta_{1} \cosh \theta_{1}\left(x_{b}-t_{0}\right) \tanh \theta_{2}\left(1-x_{b}\right)}{\theta_{2} \cosh \theta_{1}\left(x_{b}-t_{0}\right)+\theta_{1} \sinh \theta_{1}\left(x_{b}-t_{0}\right) \tanh \theta_{2}\left(1-x_{b}\right)}
$$

Using elementary algebra, including addition theorems, it is easy to see that this is equivalent to

$$
\frac{1}{\theta_{1}} \tanh \theta_{1}\left(2 t_{0}+1-x_{b}\right)+\frac{1}{\theta_{2}} \tanh \theta_{2}\left(x_{b}-1\right)=0
$$

which is equivalent to (3.3). From (12.8), we get

$$
-1-x_{b}<2 t_{0}+1-x_{b}<1+x_{b}
$$

So a necessary condition for solvability of (3.3) is

$$
\frac{1}{\theta_{1}} \tanh \left(\theta_{1}\left(1+x_{b}\right)\right)>\frac{1}{\theta_{2}} \tanh \left(\theta_{2}\left(1-x_{b}\right)\right)
$$

which is the same as inequality (3.1). Assuming condition (3.1), there exists a unique position $t_{0}$ solving (3.3). This follows by elementary considerations, utilizing the continuity and monotonicity of the respective functions. 
Now it can be shown rigorously that an interior spike located at $t^{\epsilon}$ with $t^{\epsilon} \rightarrow t_{0}$ as $\epsilon \rightarrow 0$ does exist if $\epsilon$ is small enough. The proof uses Liapunov-Schmidt reduction and follows the arguments given in [34]. We omit the technical details.

\section{Appendix E: the proof of stability of the interior spike}

Proof of Theorem 3.3: In this appendix we prove the stability of the interior spike.

We need to analyze the following eigenvalue problem

$$
\tilde{L}_{\epsilon, t^{\epsilon}} \phi_{\epsilon}=\epsilon^{2} \Delta \phi_{\epsilon}-\phi_{\epsilon}+\frac{2 A \phi_{\epsilon}}{T[A]}-\frac{A^{2}}{(T[A])^{2}}\left(T^{\prime}[A] \phi_{\epsilon}\right)=\lambda_{\epsilon} \phi_{\epsilon}
$$

where $\lambda_{\epsilon}$ is some complex number, $A=w_{\epsilon, t^{\epsilon}}+\phi_{\epsilon, t^{\epsilon}}$ with $t^{\epsilon} \in B_{\epsilon^{3 / 4}}\left(t_{0}\right)$ determined in the previous section and

$$
\phi_{\epsilon} \in H_{N}^{2}(\Omega)
$$

(Recall that $T[A]$ and $T^{\prime}[A] \phi_{\epsilon}$ have been defined in (4.10) and (11.1) respectively.)

We first study the eigenvalues with $\lambda_{\epsilon} \rightarrow \lambda_{0} \neq 0$ as $\epsilon \rightarrow 0$. The key ingredient is Theorem 2.1.

Because we study the large eigenvalues, there exists some small $c>0$ such that $\left|\lambda_{\epsilon}\right| \geq c>0$ for $\epsilon$ sufficiently small. We are looking for a condition under which $\operatorname{Re}\left(\lambda_{\epsilon}\right)<0$ for all eigenvalues $\lambda_{\epsilon}$ of $(13.1)$, (13.2) if $\epsilon$ is sufficiently small. If $\operatorname{Re}\left(\lambda_{\epsilon}\right) \leq-c$, then $\lambda_{\epsilon}$ is a stable large eigenvalue. Therefore, for the rest of this section we assume that $\operatorname{Re}\left(\lambda_{\epsilon}\right) \geq-c$ and study the stability properties of such eigenvalues.

In (13.1), (13.2) it is assumed that $\tau=0$. By a straight-forward perturbation argument all the results also hold true for $0<\tau<\tau_{0}$, for some sufficiently small $\tau_{0}>0$ which can be chosen independent of $\epsilon$.

We first rigorously derive the limit problem of (13.1), (13.2) as $\epsilon \rightarrow 0$ which will be given by a system of NLEPs. Let us assume that

$$
\left\|\phi_{\epsilon}\right\|_{H^{2}\left(\Omega_{\epsilon}\right)}=1 .
$$

We cut off $\phi_{\epsilon}$ as follows: introduce

$$
\phi_{\epsilon, 0}(y)=\phi_{\epsilon}(y) \chi\left(\frac{\epsilon y-t^{\epsilon}}{r_{0}}\right),
$$

where $\mathbf{y}=\mathbf{x} / \epsilon$ for $x \in \Omega$. 
From (13.1), (13.2), using $\operatorname{Re}\left(\lambda_{\epsilon}\right) \geq-c$ and $\left\|\phi_{\epsilon, t^{\epsilon}}\right\|_{H^{2}\left(\Omega_{\epsilon}\right)}=O(\epsilon)$, it follows that

$$
\phi_{\epsilon}=\phi_{\epsilon, 0}+O(\epsilon) \quad \text { in } H^{2}\left(\Omega_{\epsilon}\right) \text {. }
$$

Then, by a standard procedure, we extend $\phi_{\epsilon, 0}$ to a function defined on $R$ such that

$$
\left\|\phi_{\epsilon, 0}\right\|_{H^{2}(R)} \leq C\left\|\phi_{\epsilon, 0}\right\|_{H^{2}\left(\Omega_{\epsilon}\right)} .
$$

Since $\left\|\phi_{\epsilon}\right\|_{H^{2}\left(\Omega_{\epsilon}\right)}=1,\left\|\phi_{\epsilon, 0}\right\|_{H^{2}\left(\Omega_{\epsilon}\right)} \leq C$. By taking a subsequence of $\epsilon$, we may also assume that $\phi_{\epsilon, 0} \rightarrow \phi_{0}$ as $\epsilon \rightarrow 0$ in $H^{2}(R)$.

Sending $\epsilon \rightarrow 0$ with $\lambda_{\epsilon} \rightarrow \lambda_{0}$ and $x \in B_{\epsilon^{3 / 4}}\left(t_{0}\right)$, (13.1) implies

$$
\Delta \phi_{0}-\phi_{0}+2 w \phi_{0}-2 \frac{\int_{R} w \phi_{0} d y}{\int_{R} w^{2} d y} w^{2}=\lambda_{0} \phi_{0}
$$

The following result states that the stability for $\epsilon$ small enough is the same as the stability for $\epsilon=0$. Both directions are given by parts (1) and (2) of the theorem respectively. We have

Theorem 13.1. Let $\lambda_{\epsilon}$ be an eigenvalue of (13.1) and (13.2) such that $\operatorname{Re}\left(\lambda_{\epsilon}\right)>-c$ for some $c>0$.

(1) Suppose that (for suitable sequences $\epsilon_{n} \rightarrow 0$ ) we have $\lambda_{\epsilon_{n}} \rightarrow \lambda_{0} \neq 0$. Then $\lambda_{0}$ is an eigenvalue of the problem (NLEP) given in (13.5).

(2) Let $\lambda_{0} \neq 0$ with $\operatorname{Re}\left(\lambda_{0}\right)>0$ be an eigenvalue of the problem (NLEP) given in (13.5). Then, for $\epsilon$ sufficiently small, there is an eigenvalue $\lambda_{\epsilon}$ of (13.1) and (13.2) such that $\lambda_{\epsilon} \rightarrow \lambda_{0}$ as $\epsilon \rightarrow 0$.

Proof: (1) of Theorem 13.1 follows by asymptotic analysis similar to Appendix C.

To prove (2) of Theorem 13.1, we use an argument of Dancer given in Section 2 of [8]. For details on how to transfer his argument to the Gierer-Meinhardt system, we refer to [33] where it is applied to a related problem.

We now study the stability of (13.1), (13.2).

We can apply Theorem 2.1 (2) with $\gamma=2$ to (13.5). This implies that

$$
\operatorname{Re}\left(\lambda_{0}\right) \leq c_{0}<0 \quad \text { for some } c_{0}>0
$$

By Theorem 13.1 (1), all eigenvalues $\lambda_{\epsilon}$ of (13.1), (13.2), for which $\left|\lambda_{\epsilon}\right| \geq c>0$ holds, satisfy $\operatorname{Re}\left(\lambda_{\epsilon}\right) \leq$ $-c<0$ for $\epsilon$ sufficiently small. This implies that $A=w_{\epsilon, t^{\epsilon}}+\phi_{\epsilon, t^{\epsilon}}$ is linearly stable. 
Now we investigate the small eigenvalues of the problem (13.1), (13.2), i.e. we assume that $\lambda_{\epsilon} \rightarrow 0$ as $\epsilon \rightarrow 0$.

For $\epsilon$ small enough, let

$$
\bar{w}_{\epsilon}=\xi_{\epsilon}^{-1}\left[w_{\epsilon, t^{\epsilon}}+\phi_{\epsilon, t^{\epsilon}}\right], \quad \bar{h}_{\epsilon}=T\left[w_{\epsilon, t^{\epsilon}}+\phi_{\epsilon, t^{\epsilon}}\right],
$$

where $t^{\epsilon}$ is the point which has been determined by Liapunov-Schmidt reduction.

After re-scaling, the eigenvalue problem (13.1), (13.2) becomes

$$
\left\{\begin{array}{c}
\epsilon^{2} \Delta \phi_{\epsilon}-\phi_{\epsilon}+2 \frac{\bar{w}_{\epsilon}}{\bar{h}_{\epsilon}} \phi_{\epsilon}-\frac{\bar{w}_{\epsilon}^{2}}{\bar{h}_{\epsilon}^{2}} \psi_{\epsilon}=\lambda_{\epsilon} \phi_{\epsilon}, \\
\left(D(x) \psi_{\epsilon, x}\right)_{x}-\psi_{\epsilon}+2 \xi_{\epsilon} \bar{w}_{\epsilon} \phi_{\epsilon}=\lambda_{\epsilon} \tau \psi_{\epsilon},
\end{array}\right.
$$

where $\xi_{\epsilon}=\frac{1}{6 \epsilon}$ is given by $(4.8)$.

Our basic idea is the following: the eigenfunction $\phi_{\epsilon}$ can be represented as

$$
\left.a_{0}^{\epsilon} \frac{d}{d t}\left(w_{\epsilon, t}\right)\right|_{t=t^{\epsilon}}
$$

However, there is the following difficulty: note that $w_{\epsilon, t} \sim \xi_{0}(t) w\left(\frac{x-t}{\epsilon}\right) \chi\left(\frac{x-t}{r_{0}}\right)$. So when we differentiate $w_{\epsilon, t}$ with respect to $t$, we also need to differentiate $\xi_{0}(t)$ with respect to $t$.

Let us define

$$
\tilde{w}_{\epsilon, 0}(x)=\chi\left(\frac{x-t^{\epsilon}}{r_{0}}\right) \bar{w}_{\epsilon}(x)
$$

where $r_{0}$ and $\chi(x)$ are given in (4.2) and (4.3) respectively. Similar to Appendix C, we define

$$
\begin{aligned}
& \mathcal{K}_{\epsilon, t^{\epsilon}}^{\text {new }}:=\operatorname{span}\left\{\tilde{w}_{\epsilon, 0}^{\prime}\right\} \subset H^{2}\left(\Omega_{\epsilon}\right), \\
& \mathcal{C}_{\epsilon, t^{\epsilon}}^{\text {new }}:=\operatorname{span}\left\{\tilde{w}_{\epsilon, 0}^{\prime}\right\} \subset L^{2}\left(\Omega_{\epsilon}\right) .
\end{aligned}
$$

Then it is easy to see that

$$
\bar{w}_{\epsilon}(x)=\tilde{w}_{\epsilon, 0}(x)+\text { e.s.t. }
$$

and

$$
\begin{gathered}
\bar{h}_{\epsilon}^{\prime}\left(t_{0}\right)=-\xi_{\epsilon} \int_{-1}^{1} \nabla_{t_{0}} H\left(t_{0}, z\right) \bar{w}_{\epsilon}^{2} d z \\
=-\left[\left.\nabla_{x} H\left(x, t_{0}\right)\right|_{x=t_{0}}\right] \hat{\xi}_{0}^{2}+O(\epsilon)=O(\epsilon)
\end{gathered}
$$

by $(12.7)$. 
Note that $\tilde{w}_{\epsilon, 0}(x)=\hat{\xi}_{0} \tilde{w}_{0}(x)+O(\epsilon)$ in $H_{\epsilon}^{2}(-1,1)$ and $\tilde{w}_{\epsilon, 0}$ satisfies

$$
\epsilon^{2} \Delta \tilde{w}_{\epsilon, 0}-\tilde{w}_{\epsilon, 0}+\frac{\left(\tilde{w}_{\epsilon, 0}\right)^{2}}{\bar{h}_{\epsilon}}+\text { e.s.t. }=0 \text {. }
$$

Thus $\tilde{w}_{\epsilon, 0}^{\prime}:=\frac{d \tilde{w}_{\epsilon, 0}}{d x}$ satisfies

$$
\epsilon^{2} \Delta \tilde{w}_{\epsilon, 0}^{\prime}-\tilde{w}_{\epsilon, 0}^{\prime}+\frac{2 \tilde{w}_{\epsilon, 0}}{\bar{h}_{\epsilon}} \tilde{w}_{\epsilon, 0}^{\prime}-\frac{\tilde{w}_{\epsilon, 0}^{2}}{\left(\bar{h}_{\epsilon}\right)^{2}} \bar{h}_{\epsilon}^{\prime}+\text { e.s.t. }=0 .
$$

Let us now decompose

$$
\phi_{\epsilon}=\epsilon a_{0}^{\epsilon} \tilde{w}_{\epsilon, 0}^{\prime}+\phi_{\epsilon}^{\perp}
$$

with complex numbers $a_{0}^{\epsilon}$. (The scaling factor $\epsilon$ is introduced to ensure $\phi_{\epsilon}=O(1)$ in $H^{2}\left(\Omega_{\epsilon}\right)$ ), where $\phi_{\epsilon}^{\perp} \perp \mathcal{K}_{\epsilon, t^{\epsilon}}^{n e w}$.

Suppose that $\left\|\phi_{\epsilon}\right\|_{H^{2}\left(\Omega_{\epsilon}\right)}=1$. Then $\left|a_{j}^{\epsilon}\right| \leq C$.

The decomposition of $\phi_{\epsilon}$ implies the following decomposition of $\psi_{\epsilon}$ :

$$
\psi_{\epsilon}=\epsilon a_{0}^{\epsilon} \psi_{\epsilon, 0}+\psi_{\epsilon}^{\perp}, \quad \psi_{\epsilon, 0}, \psi_{\epsilon}^{\perp} \in H_{N}^{2}\left(\Omega_{\epsilon}\right)
$$

where $\psi_{\epsilon, 0}$ satisfies

$$
\left(D(x) \psi_{\epsilon, 0, x}\right)_{x}-\psi_{\epsilon, 0}+2 \xi_{\epsilon} \bar{w}_{\epsilon} \tilde{w}_{\epsilon, 0}^{\prime}=0
$$

and $\psi_{\epsilon}^{\perp}$ is given by

$$
\left(D(x) \psi_{\epsilon, x}^{\perp}\right)_{x}-\psi_{\epsilon}^{\perp}+2 \xi_{\epsilon} \bar{w}_{\epsilon} \phi_{\epsilon}^{\perp}=0 .
$$

Substituting the decompositions of $\phi_{\epsilon}$ and $\psi_{\epsilon}$ into (13.7), we have, using (13.11),

$$
\begin{gathered}
\epsilon a_{0}^{\epsilon}\left(\frac{\left(\tilde{w}_{\epsilon, 0}\right)^{2}}{\bar{h}_{\epsilon}^{2}} \bar{h}_{\epsilon}^{\prime}-\frac{\left(\bar{w}_{\epsilon}\right)^{2}}{\bar{h}_{\epsilon}^{2}} \psi_{\epsilon, 0}\right) \\
+\epsilon^{2} \Delta \phi_{\epsilon}^{\perp}-\phi_{\epsilon}^{\perp}+2 \bar{w}_{\epsilon} \bar{h}_{\epsilon} \phi_{\epsilon}^{\perp}-\frac{\bar{w}_{\epsilon}^{2}}{\bar{h}_{\epsilon}^{2}} \psi_{\epsilon}^{\perp}-\lambda_{\epsilon} \phi_{\epsilon}^{\perp}+\text { e.s.t. }=\lambda_{\epsilon} \epsilon a_{0}^{\epsilon} \tilde{w}_{\epsilon, 0}^{\prime} .
\end{gathered}
$$

Let us first compute

$$
\begin{aligned}
I_{4} & :=\epsilon a_{0}^{\epsilon}\left(\frac{\left(\tilde{w}_{\epsilon, 0}\right)^{2}}{\bar{h}_{\epsilon}^{2}} \bar{h}_{\epsilon}^{\prime}-\frac{\left(\bar{w}_{\epsilon}\right)^{2}}{\bar{h}_{\epsilon}^{2}} \psi_{\epsilon, 0}\right) \\
& =\epsilon a_{0}^{\epsilon} \frac{\left(\tilde{w}_{\epsilon, 0}\right)^{2}}{\bar{h}_{\epsilon}^{2}}\left[-\psi_{\epsilon, 0}+\bar{h}_{\epsilon}^{\prime}\right]+\text { e.s.t.. }
\end{aligned}
$$

Let us also put

$$
\tilde{L}_{\epsilon} \phi_{\epsilon}^{\perp}:=\epsilon^{2} \Delta \phi_{\epsilon}^{\perp}-\phi_{\epsilon}^{\perp}+\frac{2 \bar{w}_{\epsilon}}{\bar{h}_{\epsilon}} \phi_{\epsilon}^{\perp}-\frac{\bar{w}_{\epsilon}^{2}}{\bar{h}_{\epsilon}^{2}} \psi_{\epsilon}^{\perp} .
$$


Multiplying both sides of $(13.16)$ by $\tilde{w}_{\epsilon, 0}^{\prime}$ and integrating over $(-1,1)$, we obtain

$$
\begin{aligned}
\text { r.h.s. } & =\epsilon \lambda_{\epsilon} a_{0}^{\epsilon} \int_{-1}^{1} \tilde{w}_{\epsilon, 0}^{\prime} \tilde{w}_{\epsilon, 0}^{\prime} d x \\
& =\lambda_{\epsilon} a_{\mathbf{0}}^{\epsilon} \hat{\xi}_{0}^{2} \int_{R}\left(w^{\prime}(y)\right)^{2} d y(1+O(\epsilon))
\end{aligned}
$$

and

$$
\begin{aligned}
\text { l.h.s. } & =-\epsilon a_{0}^{\epsilon} \int_{-1}^{1} \frac{\tilde{w}_{\epsilon, 0}^{2}}{\bar{h}_{\epsilon}^{2}}\left[\psi_{\epsilon, 0}-\bar{h}_{\epsilon}^{\prime}\right] \tilde{w}_{\epsilon, 0}^{\prime} d x+\int_{-1}^{1} \frac{\tilde{w}_{\epsilon, 0}^{2}}{\bar{h}_{\epsilon}^{2}}\left(\bar{h}_{\epsilon}^{\prime} \phi_{\epsilon}^{\perp}\right) d x-\int_{-1}^{1} \frac{\tilde{w}_{\epsilon, 0}^{2}}{\bar{h}_{\epsilon}^{2}}\left(\psi_{\epsilon}^{\perp} \tilde{w}_{\epsilon, 0}^{\prime}\right) d x \\
& =\left(J_{1}+J_{2}+J_{3}\right)(1+O(\epsilon)) \text { by (13.11), }
\end{aligned}
$$

where $J_{i}, i=1,2,3$, are defined by the last equality.

For $J_{3}$, we decompose

$$
J_{3}=J_{4}+J_{5}
$$

where

$$
\begin{gathered}
J_{4}=-\int_{-1}^{1} \frac{\tilde{w}_{\epsilon, 0}^{2}}{\bar{h}_{\epsilon}^{2}}\left(\psi_{\epsilon}^{\perp}\left(t^{\epsilon}\right) \tilde{w}_{\epsilon, 0}^{\prime}\right) d x \\
J_{5}=-\int_{-1}^{1} \frac{\tilde{w}_{\epsilon, 0}^{2}}{\bar{h}_{\epsilon}^{2}}\left(\psi_{\epsilon}^{\perp}(x)-\psi_{\epsilon}^{\perp}\left(t^{\epsilon}\right)\right) \tilde{w}_{\epsilon, 0}^{\prime} d x .
\end{gathered}
$$

The following is the key lemma.

Lemma 13.2. We have

$$
\begin{aligned}
J_{1} & =-\epsilon^{2}\left(\frac{1}{3} \int_{R} w^{3} d y\right) \hat{\xi}_{0}^{3}\left(\nabla_{t^{\epsilon}}^{2} H\left(t^{\epsilon}, t^{\epsilon}\right)\right) a_{0}^{\epsilon}+o\left(\epsilon^{2}\right), \\
J_{2}+J_{3} & =o\left(\epsilon^{2}\right) .
\end{aligned}
$$

Proof: Lemma 13.2 follows from the following series of lemmas. We first study the asymptotic behavior of $\psi_{\epsilon, 0}$.

Lemma 13.3. We have

$$
\left(\psi_{\epsilon, 0}-\bar{h}_{\epsilon}^{\prime}\right)\left(t^{\epsilon}\right)=\hat{\xi}_{0}^{2} \nabla_{t^{\epsilon}} H\left(t^{\epsilon}, t^{\epsilon}\right)+O(\epsilon) .
$$


Proof: We compute $\psi_{\epsilon, 0}-\bar{h}_{\epsilon}^{\prime}$ near $t^{\epsilon}$ :

$$
\begin{aligned}
\bar{h}_{\epsilon}(x) & =\xi_{\epsilon} \int_{-1}^{1} G(x, z) \bar{w}_{\epsilon}^{2} d z \\
& =\xi_{\epsilon} \int_{-\infty}^{+\infty} K_{D}(|z|) \tilde{w}_{\epsilon, 0}^{2}(x+z) d z-\xi_{\epsilon} \int_{-1}^{1} H(x, z) \tilde{w}_{\epsilon, 0}^{2} d z+O(\epsilon) .
\end{aligned}
$$

So

$$
\bar{h}_{\epsilon}^{\prime}(x)=\xi_{\epsilon} \int_{-\infty}^{+\infty} K_{D}(|z|)\left(2 \tilde{w}_{\epsilon, 0}(x+z) \tilde{w}_{\epsilon, 0}^{\prime}(x+z)\right) d z-\xi_{\epsilon} \int_{-1}^{1} \nabla_{x} H(x, z) \tilde{w}_{\epsilon, 0}^{2} d z+O(\epsilon)
$$

Thus

$$
\left(\bar{h}_{\epsilon}^{\prime}-\psi_{\epsilon, 0}\right)(x)=-\xi_{\epsilon} \int_{-1}^{1} \nabla_{x} H(x, z) \tilde{w}_{\epsilon, 0}^{2}(z) d z-\left(-2 \xi_{\epsilon} \int_{-1}^{1} H(x, z) \tilde{w}_{\epsilon, 0} \tilde{w}_{\epsilon, 0}^{\prime} d z\right)+O(\epsilon) .
$$

Therefore,

$$
\begin{aligned}
\left(\bar{h}_{\epsilon}^{\prime}-\psi_{\epsilon, 0}\right)\left(t^{\epsilon}\right) & =-\xi_{\epsilon} \int_{-1}^{1} \nabla_{t^{\epsilon}} H\left(t^{\epsilon}, z\right) \tilde{w}_{\epsilon, 0}^{2}(\mathbf{z}) \mathbf{d z}-\left.\nabla_{z}\right|_{z=t^{\epsilon}} H\left(t^{\epsilon}, z\right) \hat{\xi}_{0}^{2}+O(\epsilon) \\
& =-\left.\nabla_{z}\right|_{z=t^{\epsilon}} H\left(z, t^{\epsilon}\right) \hat{\xi}_{0}^{2}-\left.\nabla_{z}\right|_{z=t^{\epsilon}} H\left(t^{\epsilon}, z\right) \hat{\xi}_{0}^{2}+O(\epsilon) \\
& =-\nabla_{t^{\epsilon}} H\left(t^{\epsilon}, t^{\epsilon}\right) \hat{\xi}_{0}^{2}+O(\epsilon) .
\end{aligned}
$$

This implies (13.23).

Similar to the proof of Lemma 13.3, the following result is derived.

Lemma 13.4. We have

$$
\begin{gathered}
\left(\psi_{\epsilon, 0}-\bar{h}_{\epsilon}^{\prime}\right)\left(t^{\epsilon}+\epsilon y\right)-\left(\psi_{\epsilon, 0}-\bar{h}_{\epsilon}^{\prime}\right)\left(t^{\epsilon}\right) \\
=\epsilon y \nabla_{t^{\epsilon}}^{2} H\left(t^{\epsilon}, t^{\epsilon}\right) \hat{\xi}_{0}^{2}+O\left(\epsilon^{2} y^{2}\right) .
\end{gathered}
$$

Next we estimate $\phi_{\epsilon}^{\perp}$. We derive

Lemma 13.5. For $\epsilon$ sufficiently small, we have

$$
\left\|\phi_{\epsilon}^{\perp}\right\|_{H^{2}\left(\Omega_{\epsilon}\right)}=O\left(\epsilon^{2}\right)
$$

Proof: As the first step in the proof of Lemma 13.5, we obtain a relation between $\psi_{\epsilon}^{\perp}$ and $\phi_{\epsilon}^{\perp}$. Note that similar to the proof of Proposition 11.1, $\tilde{L}_{\epsilon}$ is invertible from $\left(\mathcal{K}_{\epsilon}^{\text {new }}\right)^{\perp}$ to $\left(\mathcal{C}_{\epsilon}^{\text {new }}\right)^{\perp}$ with uniformly 
bounded inverse for $\epsilon$ small enough. By (13.16), Lemma 13.3 and the fact that $\tilde{L}_{\epsilon}$ is uniformly invertible, we deduce that

$$
\left\|\phi_{\epsilon}^{\perp}\right\|_{H^{2}\left(\Omega_{\epsilon}\right)}=O(\epsilon)
$$

Let us cut off

$$
\tilde{\phi}_{\epsilon, 0}=\frac{\phi_{\epsilon}^{\perp}}{\epsilon} \chi\left(\frac{x-t^{\epsilon}}{r_{0}}\right)
$$

Then

$$
\phi_{\epsilon}^{\perp}=\epsilon \tilde{\phi}_{\epsilon, 0}+O\left(\epsilon^{2}\right)
$$

Suppose that

$$
\tilde{\phi}_{\epsilon, 0} \rightarrow \phi_{0} \text { in } H_{N, l o c}^{2}\left(\Omega_{\epsilon}\right)
$$

Then we have by the equation for $\psi_{\epsilon}^{\perp}$ :

$$
\begin{aligned}
\psi_{\epsilon}^{\perp}\left(t^{\epsilon}\right) & =2 \epsilon \xi_{\epsilon} \int_{-1}^{1} G\left(t^{\epsilon}, z\right) \bar{w}_{\epsilon} \tilde{\phi}_{\epsilon, 0} d z \\
& =2 \epsilon G\left(t^{\epsilon}, t^{\epsilon}\right) \hat{\xi}_{0} \frac{\int_{R} w \phi_{0} d y}{\int_{R} w^{2} d y}+O\left(\epsilon^{2}\right) .
\end{aligned}
$$

This relation between $\psi_{\epsilon}^{\perp}$ and $\phi_{\epsilon}^{\perp}$ will be important for the rest of the proof.

Now we substitute (13.30) into (13.16) and, using Lemma 13.3, we have that the limit $\phi_{0}$ satisfies

$$
\Delta \phi_{0}-\phi_{0}+2 w \phi_{0}-2 G\left(t_{0}, t_{0}\right) \hat{\xi}_{0} \frac{\int_{R} w \phi_{0}}{\int_{R} w^{2}} w^{2}+\nabla_{t_{0}} H\left(t_{0}, t_{0}\right) \hat{\xi}_{0}^{2} a^{0} w^{2}=0
$$

where

$$
a^{0}=\lim _{\epsilon \rightarrow 0} a_{\epsilon}
$$

Hence, using the relations

$$
G\left(t_{0}, t_{0}\right) \hat{\xi}_{0}=1, \quad \nabla_{t_{0}} H\left(t_{0}, t_{0}\right)=0
$$

we can apply Theorem $2.1(3)$ with $\gamma=2$, which implies that $\phi_{0}=0$.

Finally, we deduce

$$
\phi_{\epsilon}^{\perp}=\epsilon \tilde{\phi}_{\epsilon, 0}+O\left(\epsilon^{2}\right)=\epsilon \phi_{0}\left(\frac{x-t^{\epsilon}}{\epsilon}\right)+O\left(\epsilon^{2}\right)=\mathbf{O}\left(\epsilon^{2}\right),
$$

which implies (13.26).

By (13.32), we have $J_{2}=o\left(\epsilon^{2}\right)$. 
From Lemma 13.5 and (13.30), we have that

$$
\psi_{\epsilon}^{\perp}\left(t^{\epsilon}\right)=O\left(\epsilon^{2}\right)
$$

Further,

$$
\psi_{\epsilon}^{\perp}\left(t^{\epsilon}+\epsilon y\right)-\psi_{\epsilon}^{\perp}\left(t^{\epsilon}\right)=-2 \epsilon^{2} y \nabla_{t^{\epsilon}} H\left(t^{\epsilon}, t^{\epsilon}\right) \hat{\xi}_{0} \frac{\int_{R} w \phi_{0} d y}{\int_{R} w^{2} d y}+O\left(\epsilon^{3}\right)=O\left(\epsilon^{3}\right) .
$$

This implies $J_{4}=o\left(\epsilon^{2}\right)$ and $J_{5}=o\left(\epsilon^{2}\right)$.

The computation of $J_{1}$ follows from Lemmas 13.3 and 13.4. In fact,

$$
\begin{aligned}
J_{1}= & -\epsilon a_{0}^{\epsilon} \int_{-1}^{1} \frac{\tilde{w}_{\epsilon, 0}^{2}}{\bar{h}_{\epsilon}^{2}}\left(\psi_{\epsilon, 0}-\bar{h}_{\epsilon}^{\prime}\right) \tilde{w}_{\epsilon, 0}^{\prime} d x+o\left(\epsilon^{2}\right) \\
= & -\epsilon a_{0}^{\epsilon} \int_{-1}^{1} \frac{\tilde{w}_{\epsilon, 0}^{2}}{\bar{h}_{\epsilon}^{2}}\left(\psi_{\epsilon, 0}\left(t_{0}\right)-\bar{h}_{\epsilon}^{\prime}\left(t_{0}\right)\right) \tilde{w}_{\epsilon, 0}^{\prime} d x+o\left(\epsilon^{2}\right) \\
& -\epsilon a_{0}^{\epsilon} \int_{-1}^{1} \frac{\tilde{w}_{\epsilon, 0}^{2}}{\bar{h}_{\epsilon}^{2}}\left(\left[\psi_{\epsilon, 0}(x)-\bar{h}_{\epsilon}^{\prime}(x)\right]-\left[\psi_{\epsilon, 0}\left(t_{0}\right)-\bar{h}_{\epsilon}^{\prime}\left(t_{0}\right)\right]\right) \tilde{w}_{\epsilon, 0}^{\prime} d x+o\left(\epsilon^{2}\right) \\
= & J_{6}+J_{7} .
\end{aligned}
$$

For $J_{6}$, we use Lemma 13.3 to obtain

$$
\begin{aligned}
J_{6} & =-\frac{2}{3} \epsilon a_{0}^{\epsilon} \int_{-1}^{1} \frac{\tilde{w}_{\epsilon, 0}^{3}}{\bar{h}_{\epsilon}^{3}} \bar{h}_{\epsilon}^{\prime}\left(\psi_{\epsilon, 0}\left(t_{0}\right)-\bar{h}_{\epsilon}^{\prime}\left(t_{0}\right)\right) d x+o\left(\epsilon^{2}\right) \\
& =-\frac{2}{3} \epsilon^{2} a_{0}^{\epsilon}\left(\int_{R} w^{3} d y\right) \bar{h}_{\epsilon}^{\prime}\left(t^{\epsilon}\right) \hat{\xi}_{\mathbf{0}}^{2} \nabla_{\mathbf{t}^{\epsilon}} \mathbf{H}\left(\mathbf{t}^{\epsilon}, \mathbf{t}^{\epsilon}\right)+o\left(\epsilon^{2}\right)=o\left(\epsilon^{2}\right)
\end{aligned}
$$

since $\nabla_{\mathbf{t}^{\epsilon}} \mathbf{H}\left(\mathbf{t}^{\epsilon}, \mathbf{t}^{\epsilon}\right)=\mathbf{o}(\mathbf{1})$. Finally, using Lemma 13.4, we compute the main contribution:

$$
\begin{aligned}
J_{7} & =\epsilon^{2} \hat{\xi}_{0} \int_{R}\left(y w^{2} w^{\prime}(y)\right) d y \nabla_{t^{\epsilon}}^{2} H\left(t^{\epsilon}, t^{\epsilon}\right) \hat{\xi}_{0}^{2} a_{0}^{\epsilon}+o\left(\epsilon^{2}\right) \\
& =-\epsilon^{2}\left(\frac{1}{3} \int_{R} w^{3} d y\right) \hat{\xi}_{0}^{3} \nabla_{t^{\epsilon}}^{2} H\left(t^{\epsilon}, t^{\epsilon}\right) a_{0}^{\epsilon}+o\left(\epsilon^{2}\right) .
\end{aligned}
$$

Combining (13.35), (13.36) and using (13.10), Lemma 13.3, Lemma 13.4, we obtain (13.21).

\section{This concludes the proof of Lemma 13.2.}

Comparing (13.18) with (13.19) r.h.s. and using Lemma 13.2, (2.3), we obtain

$$
-2.4 \epsilon^{2} \hat{\xi}_{0}^{3}\left(\nabla_{t^{\epsilon}}^{2} H\left(t^{\epsilon}, t^{\epsilon}\right)\right) a_{0}^{\epsilon}+o\left(\epsilon^{2}\right)=\lambda_{\epsilon} \hat{\xi}_{0}^{2} a_{0}^{\epsilon} 1.2(1+o(1))
$$

Equation (13.37) implies that the small eigenvalue $\lambda_{\epsilon}$ of (13.7) satisfies

$$
\lambda_{\epsilon}=-2 \epsilon^{2} \hat{\xi}_{0}\left(\nabla_{t^{\epsilon}}^{2} H\left(t^{\epsilon}, t^{\epsilon}\right)\right)+o\left(\epsilon^{2}\right) .
$$


Arguing as in Theorem 13.1, this shows that since by (9.7) $\nabla_{t^{\epsilon}}^{2} H\left(t^{\epsilon}, t^{\epsilon}\right)$ is positive, the small eigenvalue $\lambda_{\epsilon}$ satisfies $\operatorname{Re}\left(\lambda_{\epsilon}\right) \leq-c \epsilon^{2}$ for some $c>0$ which is independent of $\epsilon$.

This fact, together with the results in the previous section, concludes the proof of Theorem 3.3.

\section{ACKNOWLEDGMENTS}

The work of JW is supported by a grant of RGC of Hong Kong. The work of MW is supported by a BRIEF Award of Brunel University. MW thanks the Department of Mathematics at CUHK for their kind hospitality. We thank Karen Page for a very interesting discussion on biological modeling. 


\section{REFERENCES}

[1] D. L. Benson, P. K. Maini and J. A. Sherratt, Analysis of pattern formation in reaction diffusion models with spatially inhomogeneous diffusion coefficients, Math. Comp. Mod. 17 (1993), 29-34.

[2] D. L. Benson, P. K. Maini and J. A. Sherratt, Unravelling the Turing bifurcation using spatially varying diffusion coefficients, J. Math. Biol. 37 (1998), 381-387.

[3] D. L. Benson, J. A. Sherratt and P. K. Maini, Diffusion driven instability in an inhomogeneous domain, Bull. Math. Biol. 55 (1993), 365-384.

[4] S. S. Blair, Limb development: Marginal fringe benefits, Current Biology 7 (1997), R686-R690.

[5] S. S. Blair, Developmental biology: Notching the hindbrain, Current Biology 14 (2004), R570-R572.

[6] F. Brümmer, G. Zempel, P. Buhle, J. C. Stein and D. F. Hulser, Retionic acid modulates gap junctional permeability: A comparative study of dye spreading and ionic coupling in cultured cells, Exp. Cell Res. 196 (1991), 158-163.

[7] Y.-C. Chang, M. Amoyel, X. Qiu, Y.-J. Xu and D.G. Wilkinson, Notch activation regulates the segregation and differentiation of rhombomere boundary cells in the zebrafish hindbrain, Dev. Cell 6 (2004), 539-555.

[8] E.N. Dancer, On stability and Hopf bifurcations for chemotaxis systems, Methods Appl. Anal. 8 (2001), 245-256.

[9] B. Gebelein, D. J. McKay and R. S. Mann, Direct integration of Hox and segmentation gene inputs during Drosophila development, Nature 431 (2004), 653-659.

[10] A. Gierer and H. Meinhardt, A theory of biological pattern formation, Kybernetik (Berlin) 12 (1972), 30-39.

[11] P. Gray and S.K. Scott, Autocatalytic reactions in the isothermal, continuous stirred tank reactor: isolas and other forms of multistability, Chem. Eng. Sci. 38 (1983), 29-43.

[12] P. Gray and S.K. Scott, Autocatalytic reactions in the isothermal, continuous stirred tank reactor: oscillations and instabilities in the system $A+2 B \rightarrow 3 B, B \rightarrow C$, Chem. Eng. Sci. 39 (1984), 1087-1097.

[13] D. Iron, M. J. Ward and J. Wei, The stability of spike solutions to the one-dimensional Gierer-Meinhardt model, Physica D 150 (2001), 25-62.

[14] K. D. Irvine and C. Rauskolb, Boundaries in development: Formation and function, Annu. Rev. Cell Dev. Biol. 17 (2001), 189-214. 
[15] P. K. Maini, D. L. Benson and J. A. Sherratt, Pattern formation in reaction-diffusion models with spatially inhomogeneous diffusion coefficients, IMA J. Math. Appl. Med. Biol. 9 (1992), 197-213.

[16] R. J. Major and K. D. Irvine, Localization and requirement for Myosin II at the dorsal-ventral compartment boundary of the Drosophila wing, Developmental Dynamics 235 (2006), 3051-3058.

[17] A. May, P. A. Firby and A. P. Bassom, Diffusion driven instability in an inhomogeneous circular domain, Math. Comp. Mod. 29(4) (1999), 53-66.

[18] H. Meinhardt, Cell determination boundaries as organizing regions for secondary embryonic fields, Developmental Biology 96 (1983), 375-385.

[19] J. Murray, Mathematical Biology I/II, Springer, 2002.

[20] Y. Nishiura, T. Teramoto, X. Yuan and K.-I. Ueda, Dynamics of traveling pulses in heterogeneous media, Chaos 17, (2007), 037104.

[21] K. Page, P. K. Maini and N. A. M. Monk, Pattern formation in spatially heterogeneous Turing reaction-diffusion models, Phys. D 181 (2003), 80-101.

[22] K. Page, P. K. Maini and N. A. M. Monk, Complex pattern formation in reaction-diffusion systems with spatially varying parameters, Phys. D 202 (2005), 95-115.

[23] V. M. Panin, V. Papayannopoulous, Fringe modulates Notch-ligand interactions, Nature 387 (1997), $908-912$.

[24] J. Schnakenberg, Simple chemical reaction systems with limit cycle behaviour, J. Theoret. Biol. 81 (1979), 389-400.

[25] A. M. Turing, The chemical basis of morphogenesis, Phil. Trans. Roy. Soc. Lond. B 237 (1952), 37-72.

[26] M. Ward, D. McInerney, P. Houston, D. Gavaghan and P. Maini, The dynamics and pinning of a spike for a reaction-diffusion system, SIAM J. Appl. Math. 62 (2002), 1297-1328.

[27] M.J. Ward and J. Wei, Hopf bifurcations and oscillatory instabilities of solutions for the one-dimensional GiererMeinhardt model, J. Nonlinear Sci. 13 (2003), 209-264.

[28] J. Wei, On the construction of single-peaked solutions to a singularly perturbed semilinear Dirichlet problem, J. Differential Equations 129 (1996), 315-333.

[29] J. Wei, On single interior spike solutions of Gierer-Meinhardt system: uniqueness, spectrum estimates and stability analysis, Euro. J. Appl. Math. 10 (1999), 353-378. 
[30] J. Wei, On single interior spike solutions of Gierer-Meinhardt system: uniqueness and spectrum estimates, European J. Appl. Math., 10 (1999), 353-378.

[31] J. Wei and M. Winter, On the two-dimensional Gierer-Meinhardt system with strong coupling, SIAM J. Math. Anal. 30 (1999), 1241-1263.

[32] J. Wei and M. Winter, Spikes for the two-dimensional Gierer-Meinhardt system: The strong coupling case, $J$. Differential Equations 178 (2000), 478-518.

[33] J. Wei and M. Winter, Spikes for the two-dimensional Gierer-Meinhardt system: The weak coupling case, $J$. Nonlinear Science 11 (2001), 415-458.

[34] J. Wei and M. Winter, On the Gierer-Meinhardt System with Precursors, Discrete Contin. Dyn. Syst. Ser. A, to appear.

[35] L. Wolpert, Positional information and the spatial pattern of cellular differentiation, J. Theor. Biology 25 (1969), $1-47$.

[36] L. Wolpert and A. Hornbruch, Double anterior chick limb buds and models for cartilage rudiment specification, Development 109 (1990), 961-966.

Department of Mathematics, The Chinese University of Hong Kong, Shatin, Hong Kong

E-mail address: wei@math.cuhk.edu.hk

Brunel University, Department of Mathematical Sciences, Uxbridge, UB8 3PH, United Kingdom

E-mail address: matthias.winter@brunel.ac.uk 